\title{
Synthesis of tert-Alkylamino Hydroxy Carboxylic Esters via an Intermolecular Ene-type Reaction of Oxazolones and Enol Ethers
}

\author{
Robert A. Mosey ${ }^{1}$, Jason S. Fisk ${ }^{1}$, Timothy Friebe ${ }^{2}$ and Jetze J. Tepe ${ }^{1_{*}}$ \\ ${ }^{1}$ Department of Chemistry, Michigan State University, East Lansing, Michigan 48824 \\ ${ }^{2}$ Department of Chemistry, Eastern Michigan University, Ypsilanti, Michigan, 48197
}

\section{Supporting Information (NMR Spectra)}

2-phenyl-4-carbmethoxy-5(4H)-oxazolone

2-(4-methoxyphenyl)-4-carbmethoxy-5(4H)-oxazolone

2-(4-trifluoromethylphenyl)-4-carbmethoxy-5(4H)-oxazolone

2-ethyl-4-carbmethoxy-5(4H)-oxazolone

2-benzyl-4-carbmethoxy-5(4H)-oxazolone

S5

Methyl-2-benzamido-3-tert-butoxy-2-(hydroxymethyl)butanoate (1)

Methyl-2-benzamido-3-benzyloxy-2-(hydroxymethyl)butanoate (2)

Methyl-2-benzamido-3-ethoxy-2-(hydroxymethyl)butanoate (3)

Methyl-2-(benzamido)-2-(tetrahydro-2H-pyran-2-yl)-3-hydroxypropanoate (4)

Methyl-2-(benzamido)-3-(benzyloxy)-2-(hydroxymethyl)pentanoate (5)

Methyl-2-(benzamido)-3-(methoxy)-2-(hydroxymethyl)-4-methylpentanoate (6)

Methyl-2-(benzamido)-3-butoxy-2-(hydroxymethyl)but-3-enoate (7)

Methyl-2-(benzamido)-2-(hydroxymethyl)-3-methoxy-3-methylbutanoate (8) 
2-phenyl-4-carbmethoxy-5(4H)-oxazolone
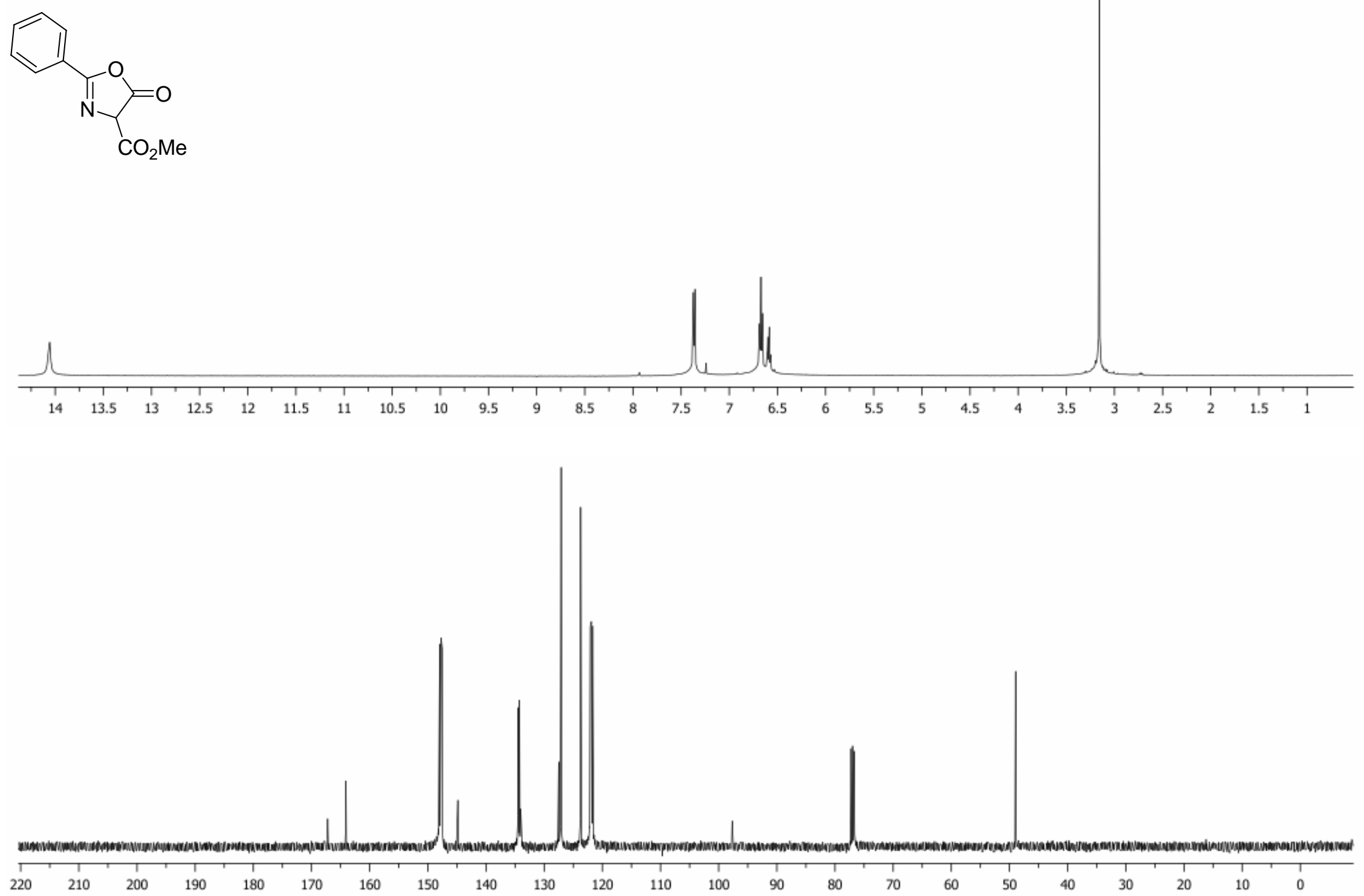
2-(4-methoxyphenyl)-4-carbmethoxy-5(4H)-oxazolone
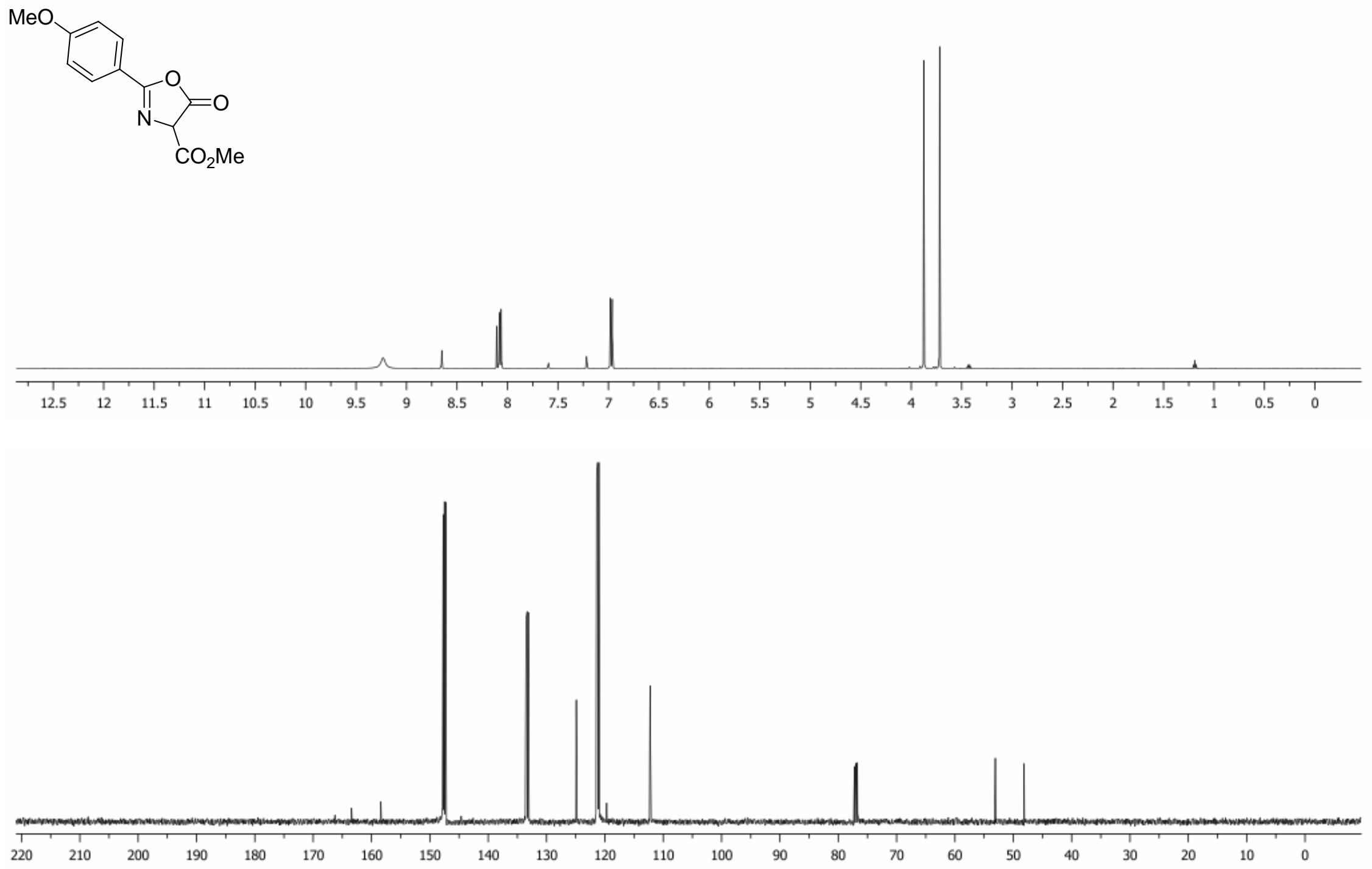
2-(4-trifluoromethylphenyl)-4-carbmethoxy-5(4H)-oxazolone
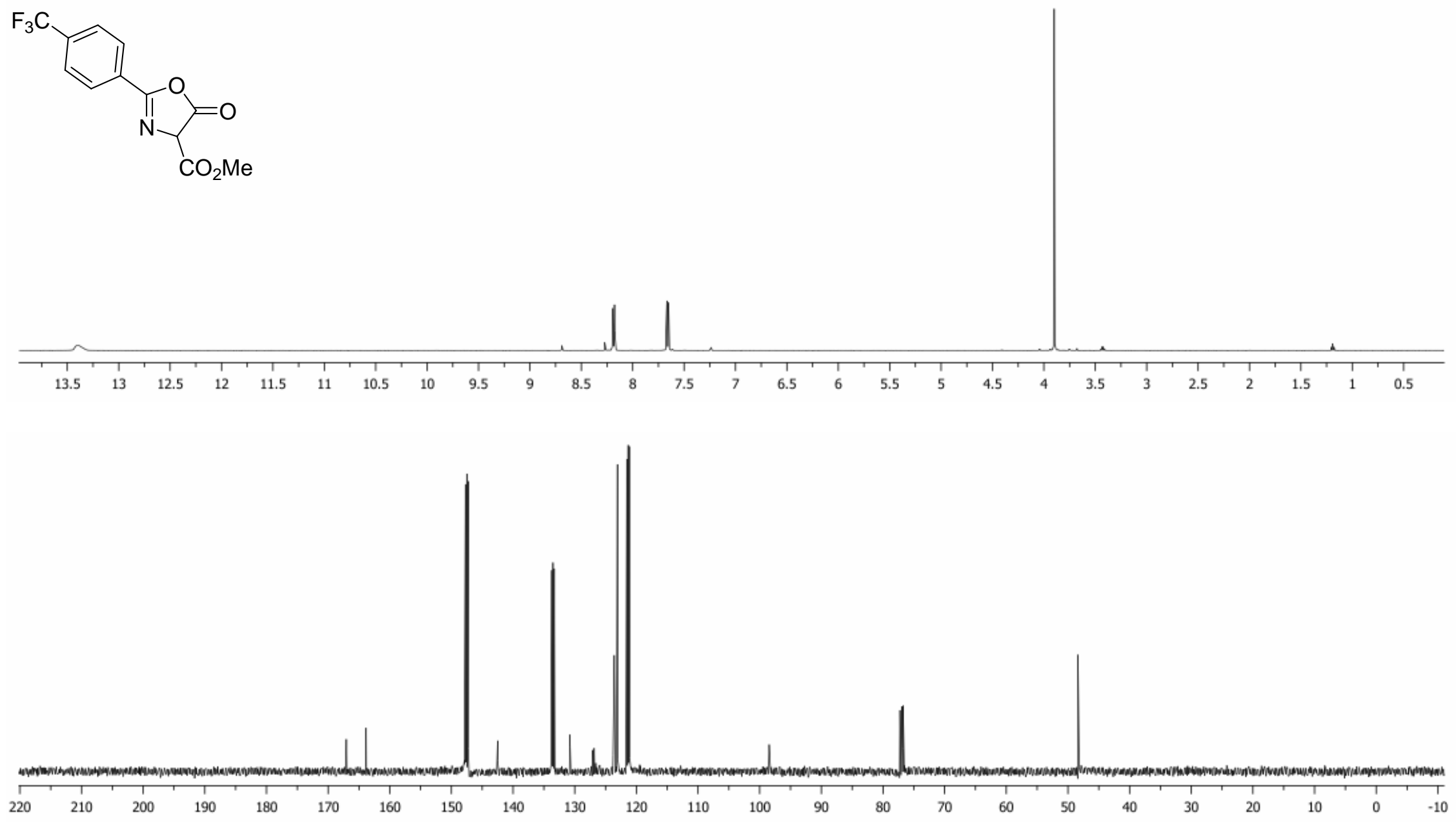
2-ethyl-4-carbmethoxy-5(4H)-oxazolone

$\mathrm{CO}_{\mathrm{CO}_{2} \mathrm{Me}}^{\mathrm{E}}$
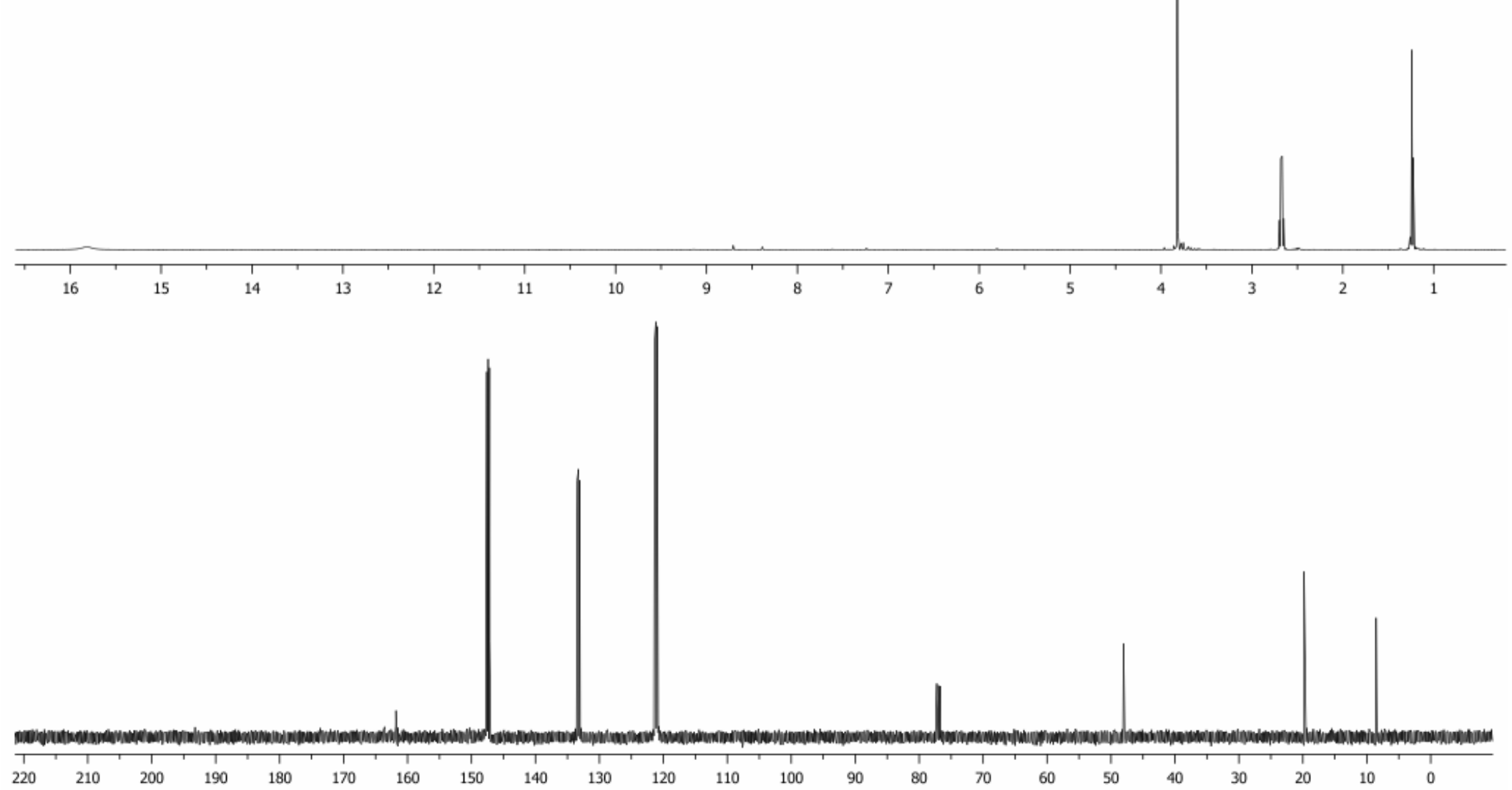

$\top$
220

$140 \quad 130$ 
2-benzyl-4-carbmethoxy-5(4H)-oxazolone

$\mathrm{CO}_{\mathrm{CO}_{2} \mathrm{Me}}^{\mathrm{Bn}}$
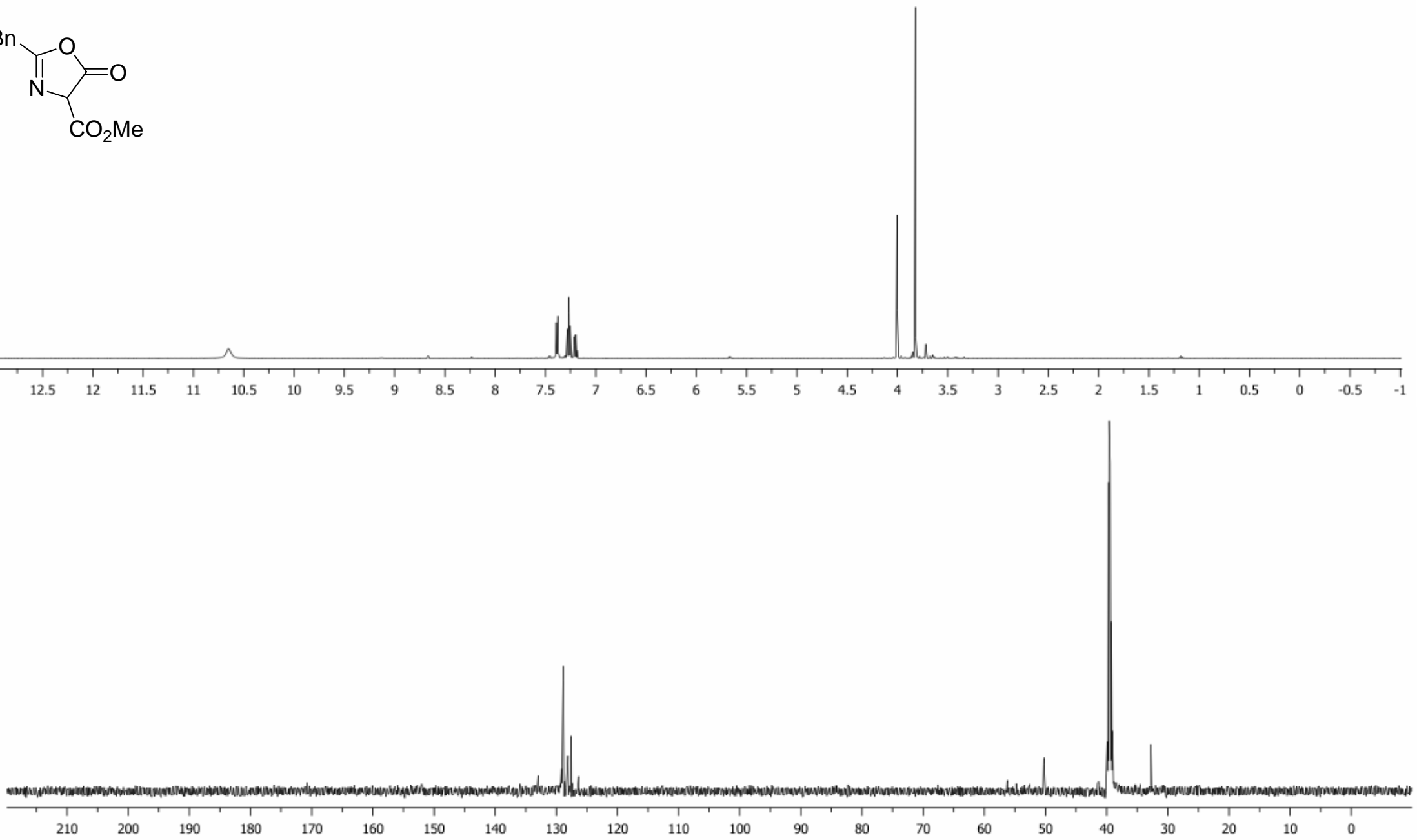
Methyl-2-benzamido-3-tert-butoxy-2-(hydroxymethyl)butanoate (1)

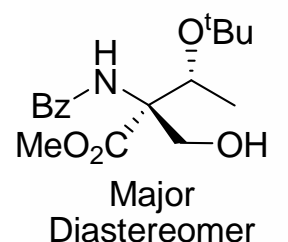

iastereomer
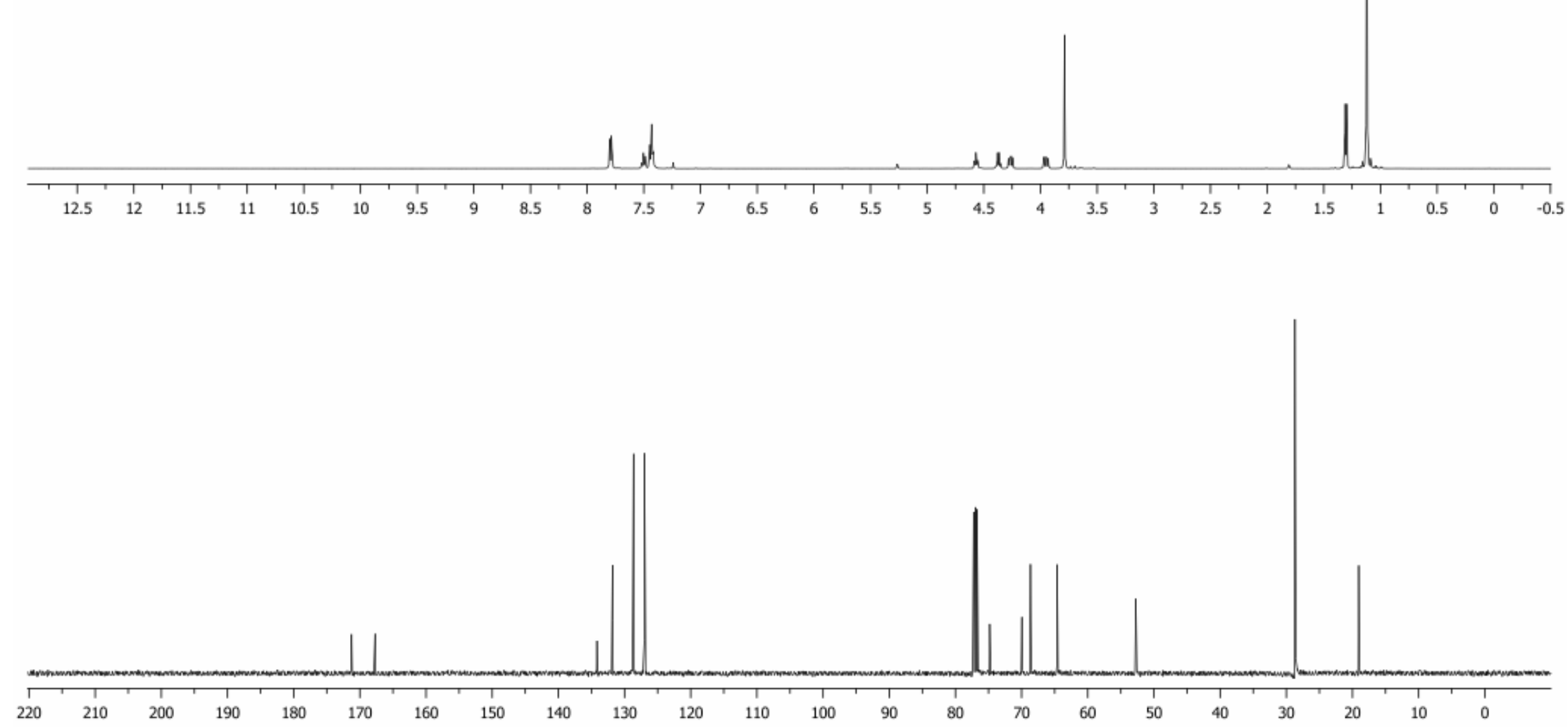
Methyl-2-benzamido-3-tert-butoxy-2-(hydroxymethyl)butanoate (1)

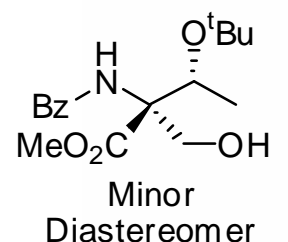

Diastereomer
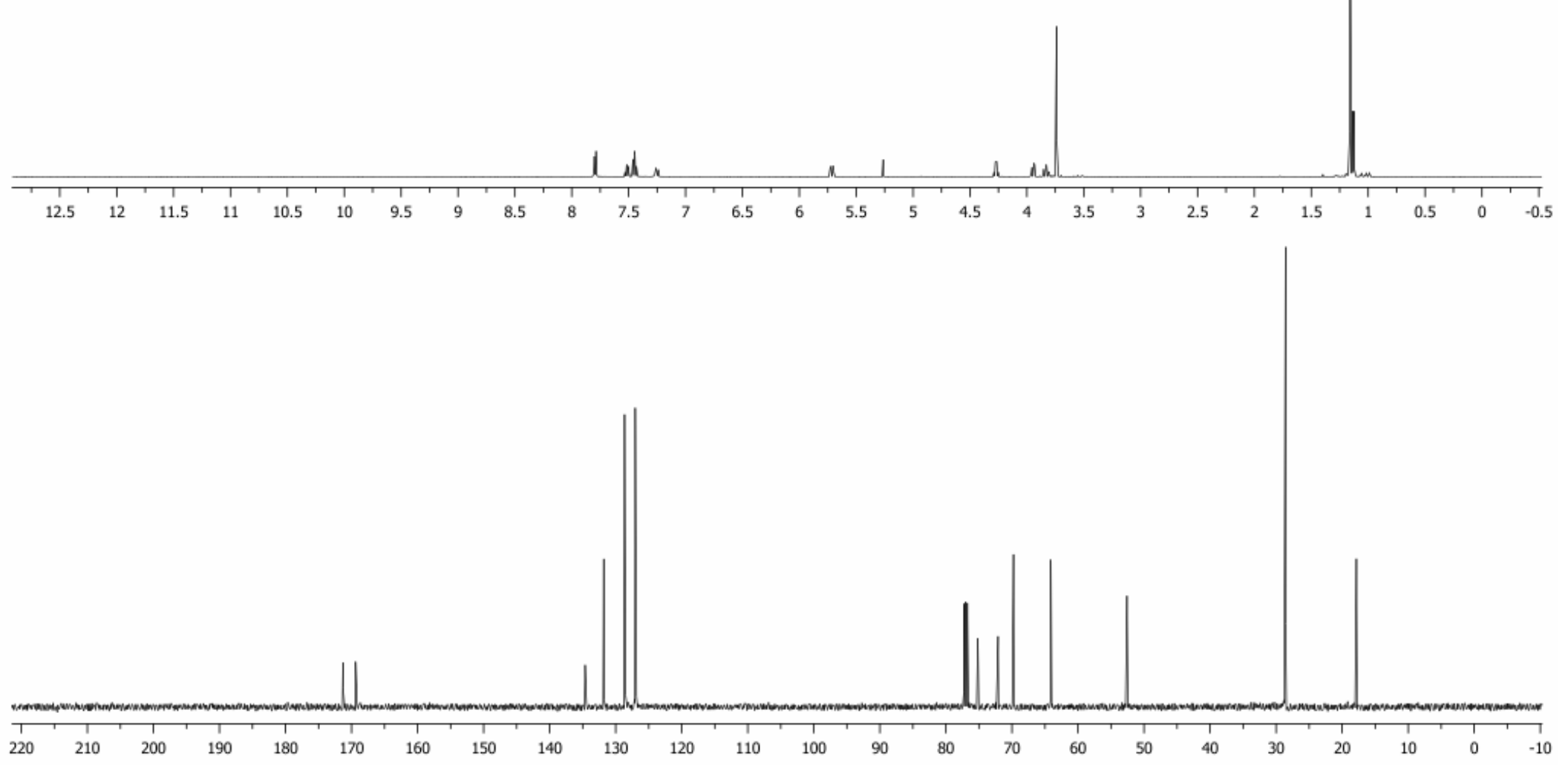
Methyl-2-benzamido-3-benzyloxy-2-(hydroxymethyl)butanoate (2)

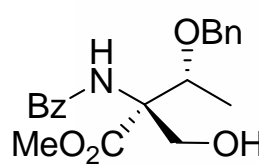

Major

Diastereomer
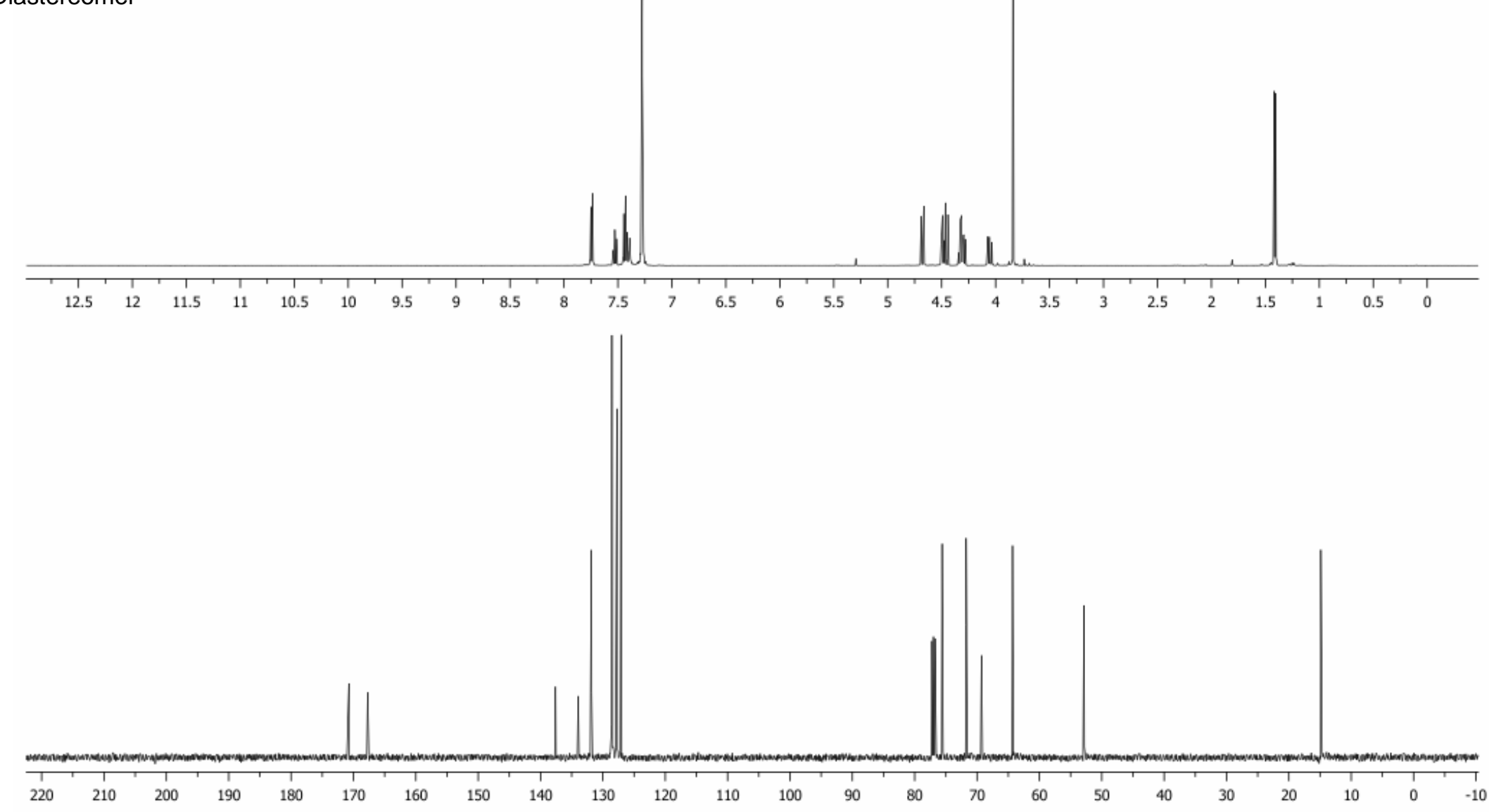
Methyl-2-benzamido-3-benzyloxy-2-(hydroxymethyl)butanoate (2)

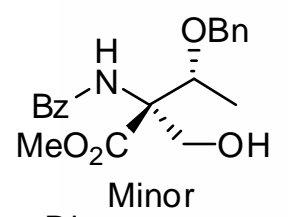

Diastereomer
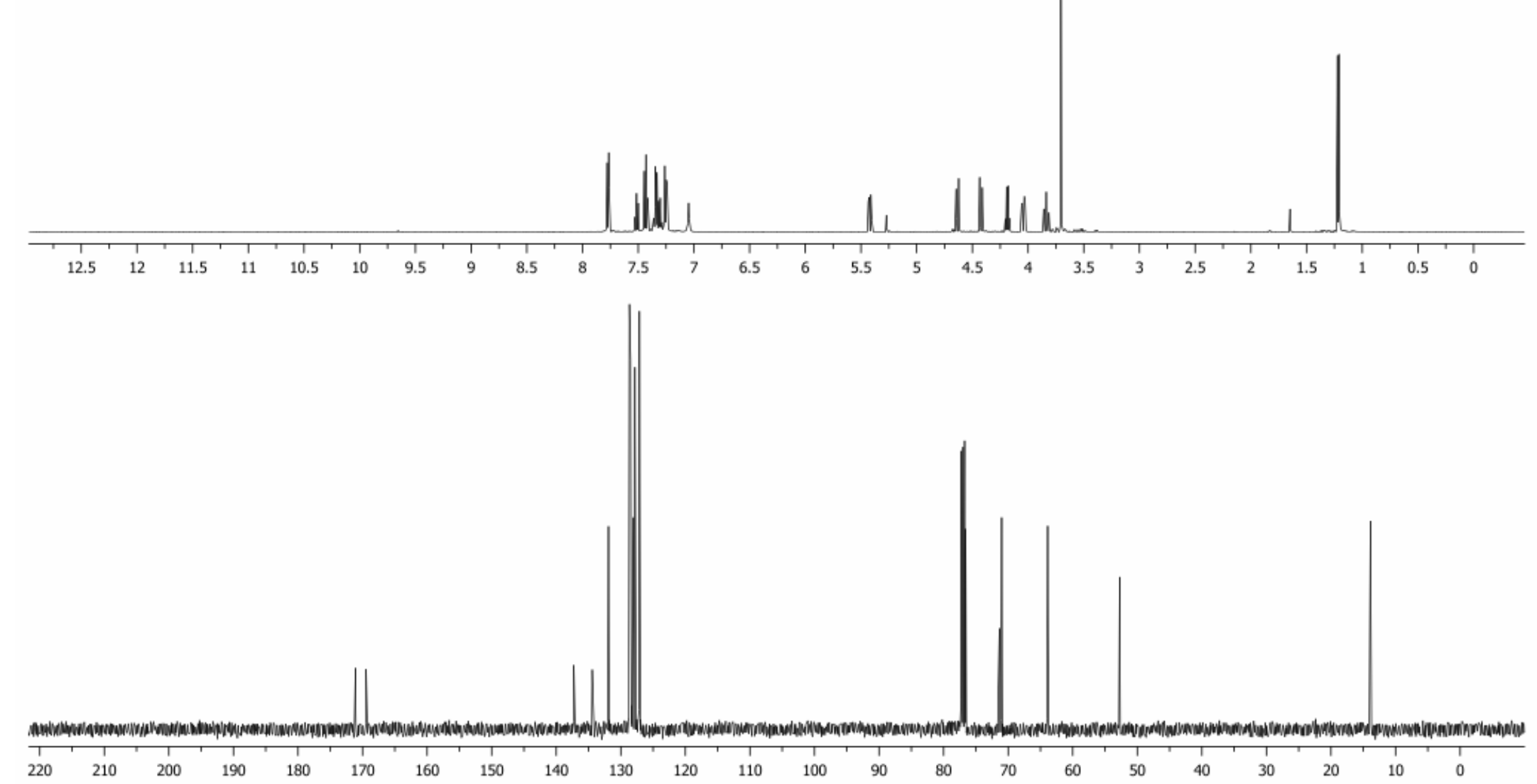
Methyl-2-benzamido-3-ethoxy-2-(hydroxymethyl)butanoate (3)

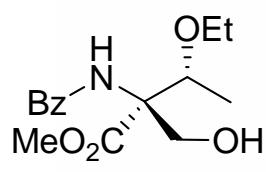

Major

Diastereomer
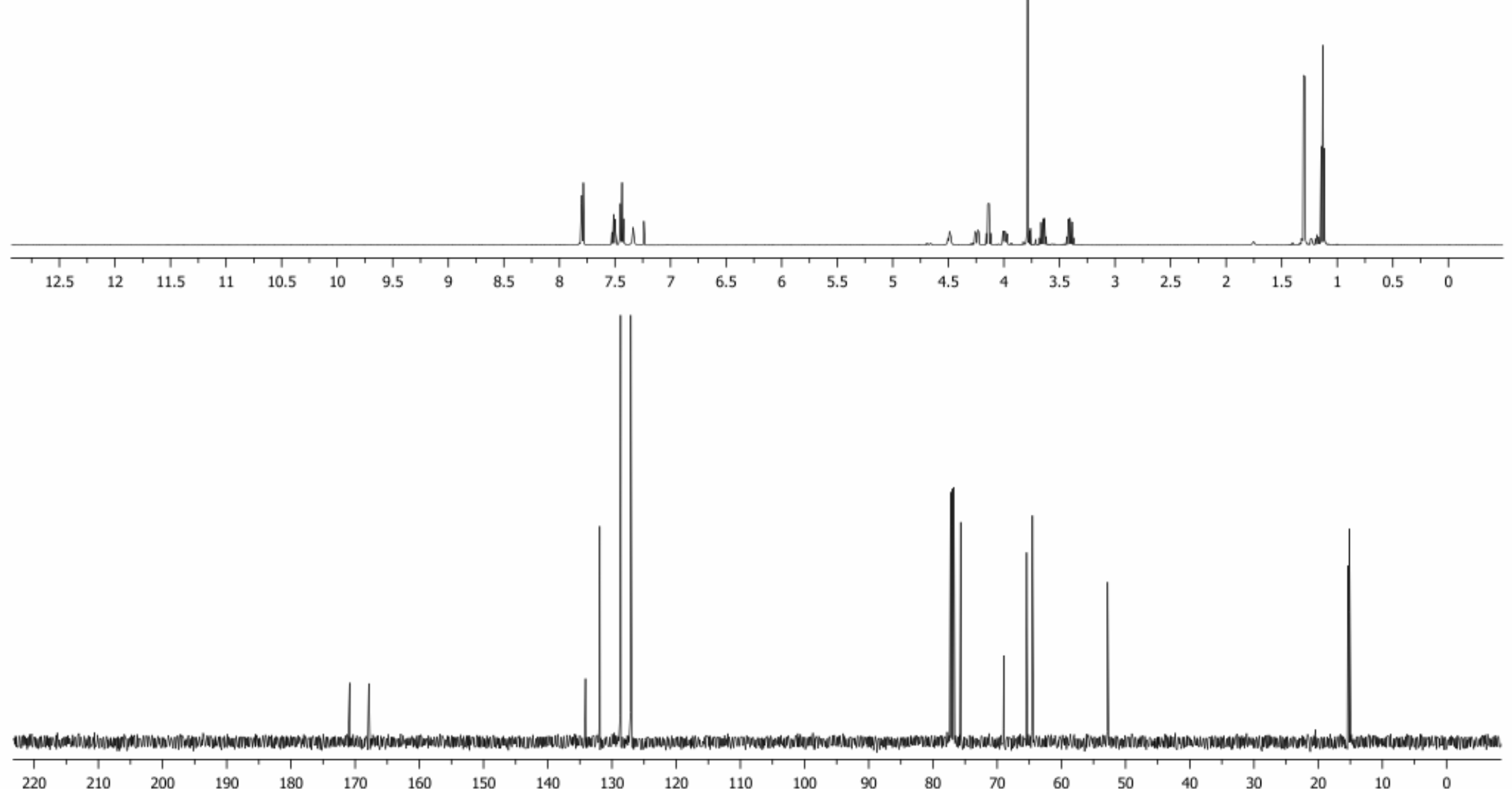
Methyl-2-benzamido-3-ethoxy-2-(hydroxymethyl)butanoate (3)

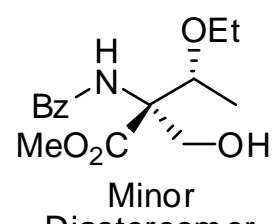

Diastereomer
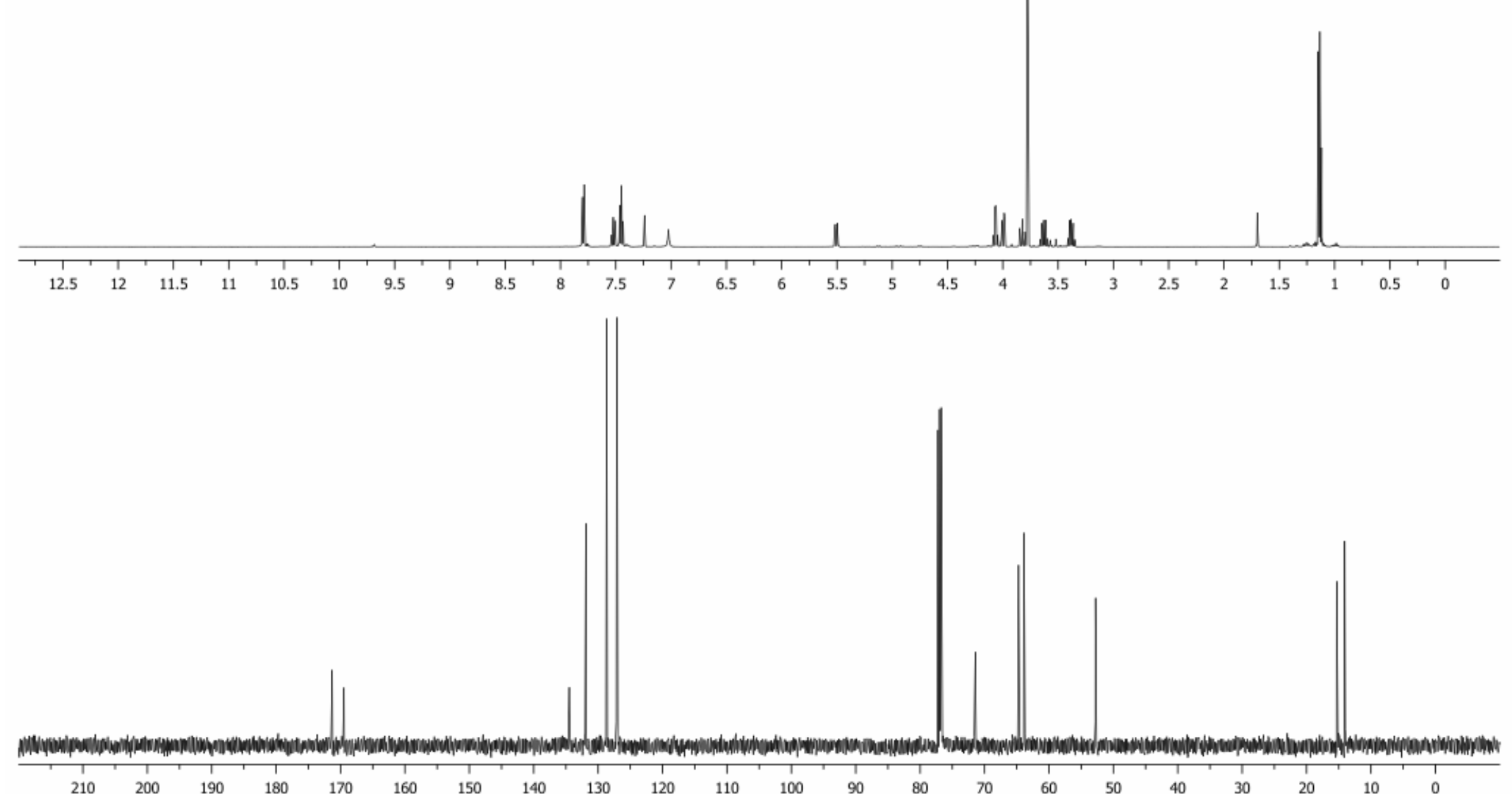
Methyl-2-(benzamido)-2-(tetrahydro-2H-pyran-2-yl)-3-hydroxypropanoate (4)

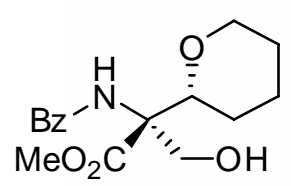

Major

Diastereomer
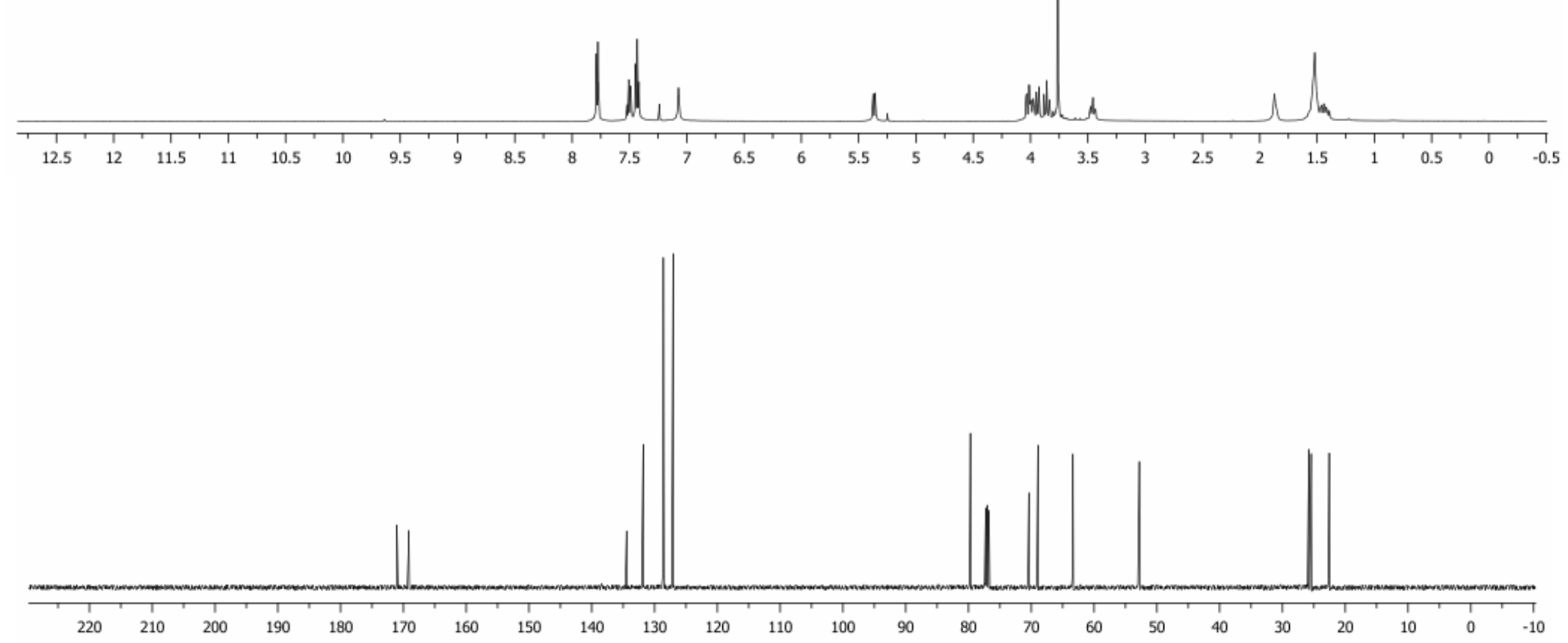
Methyl-2-(benzamido)-2-(tetrahydro-2H-pyran-2-yl)-3-hydroxypropanoate (4)

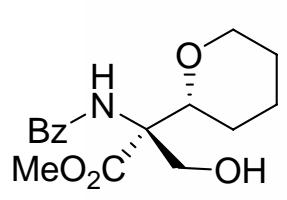

Minor

Diastereomer
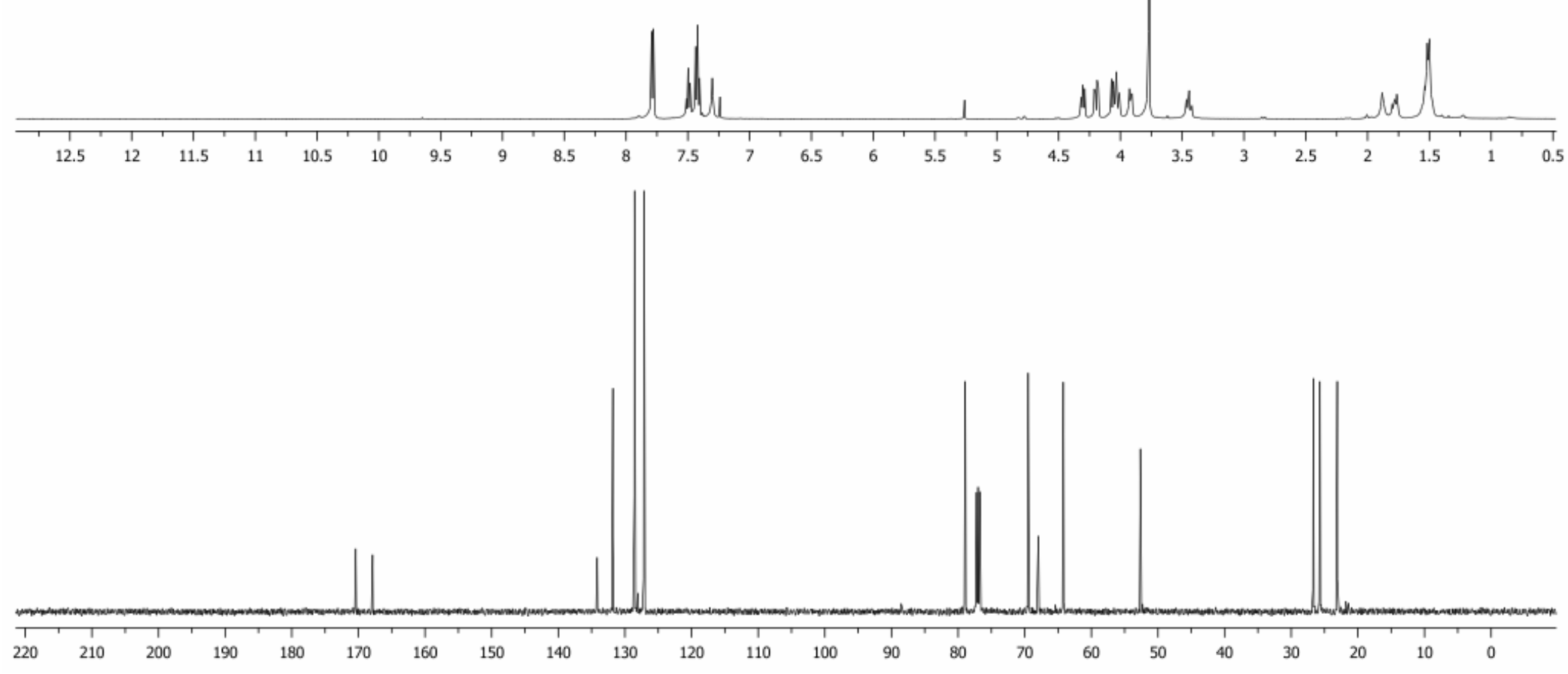
Methyl-2-(benzamido)-3-(benzyloxy)-2-(hydroxymethyl)pentanoate (5)

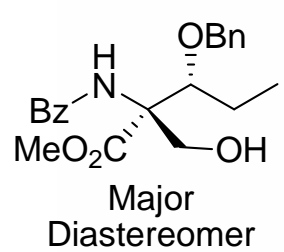

Diastereomer
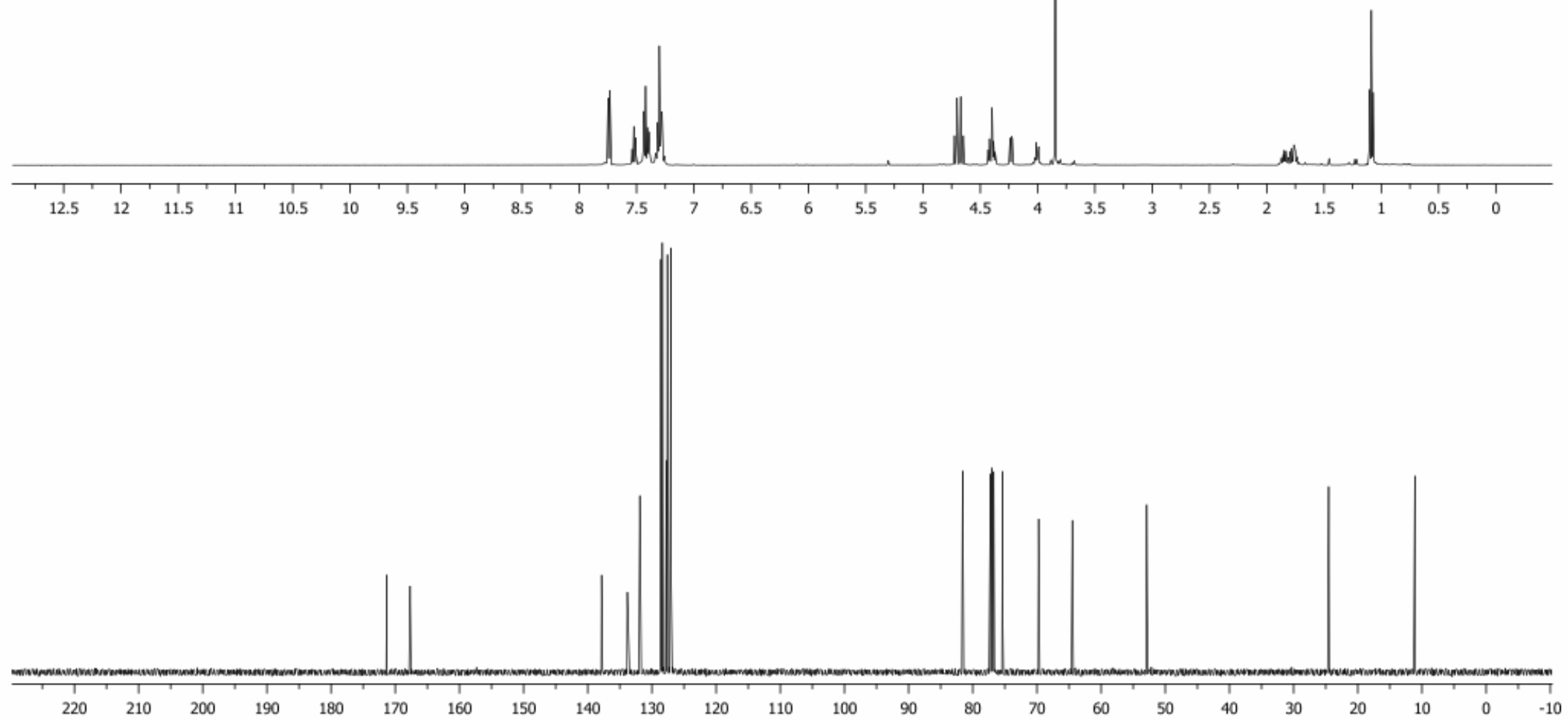
Methyl-2-(benzamido)-3-(benzyloxy)-2-(hydroxymethyl)pentanoate (5)
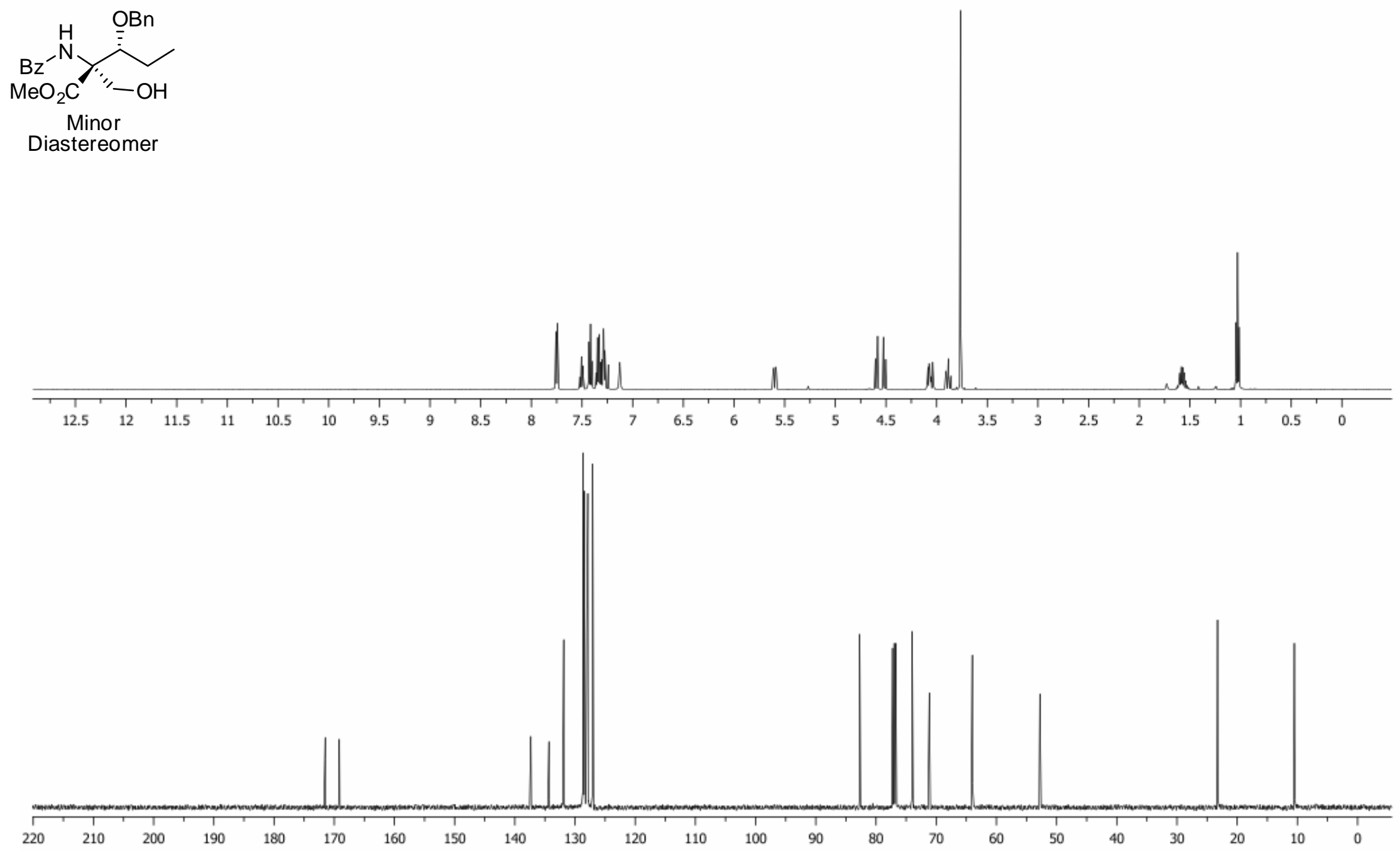
Methyl-2-(benzamido)-3-(methoxy)-2-(hydroxymethyl)-4-methylpentanoate (6)

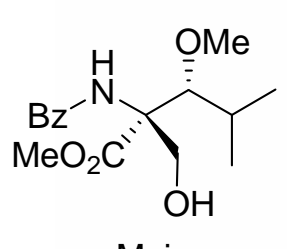

Major

Diastereomer
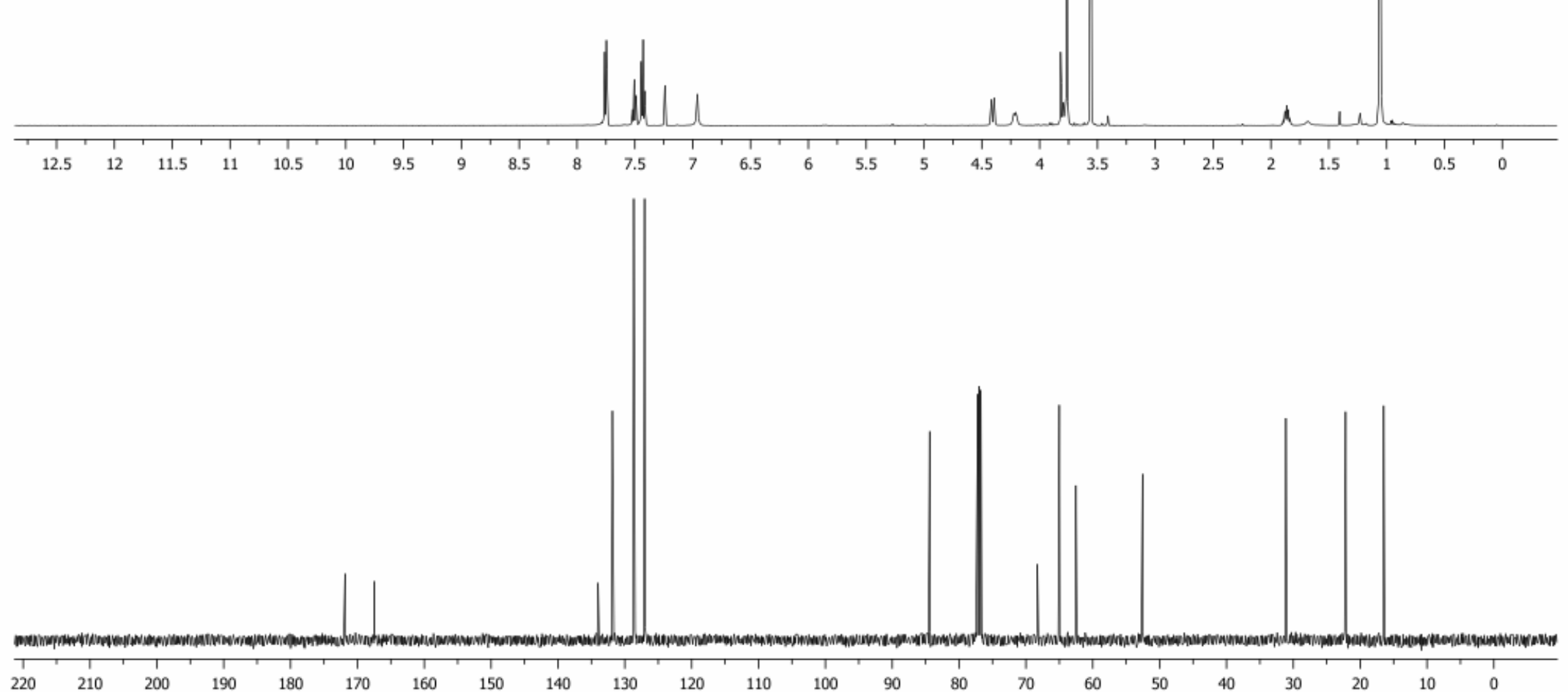
Methyl-2-(benzamido)-3-(methoxy)-2-(hydroxymethyl)-4-methylpentanoate (6)

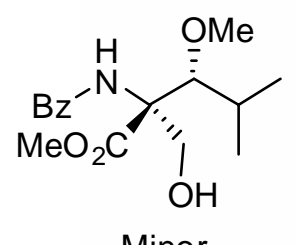

$$
\text { Mino }
$$

Diastereomer
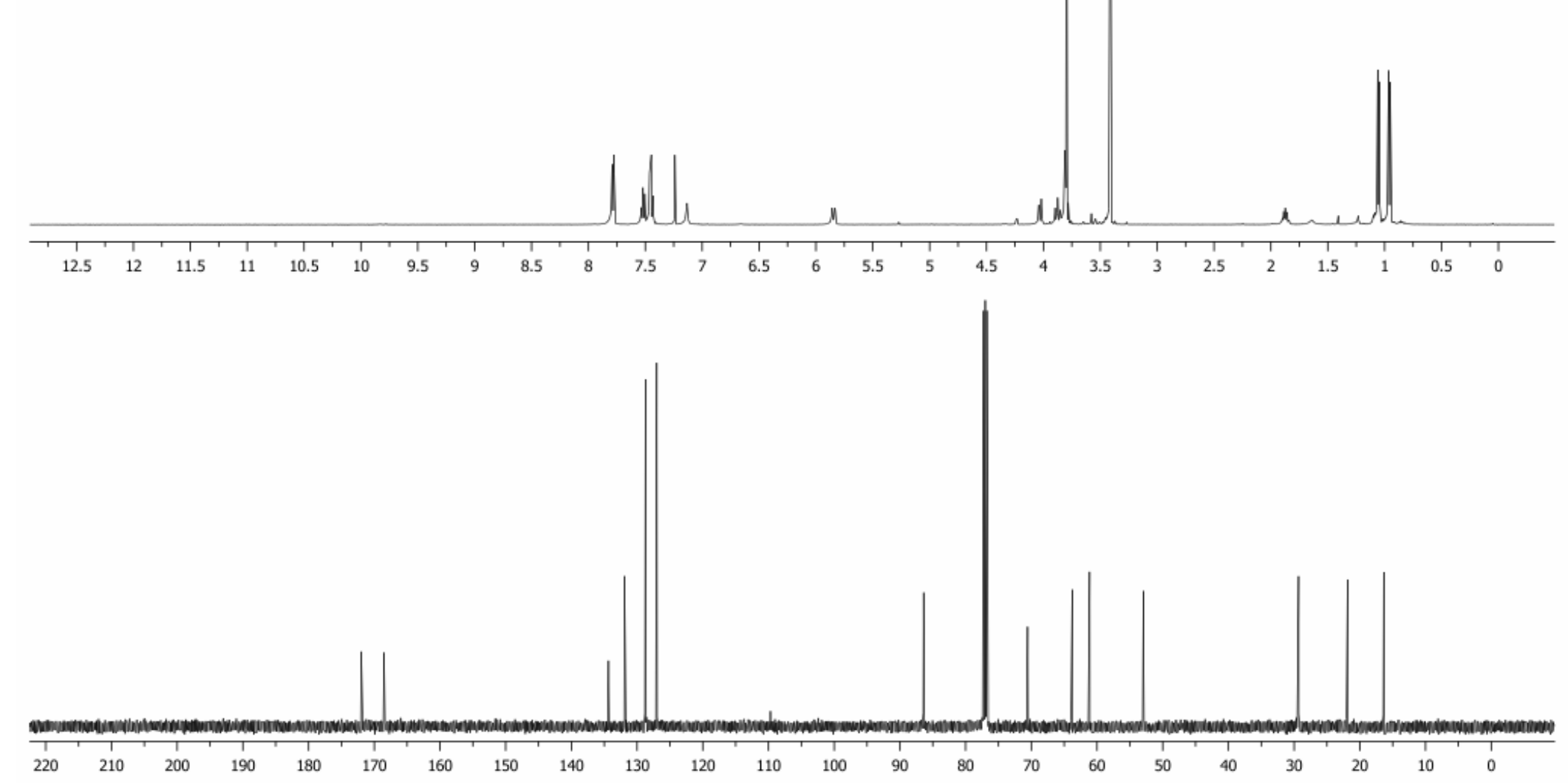
Methyl-2-(benzamido)-3-butoxy-2-(hydroxymethyl)but-3-enoate (7)
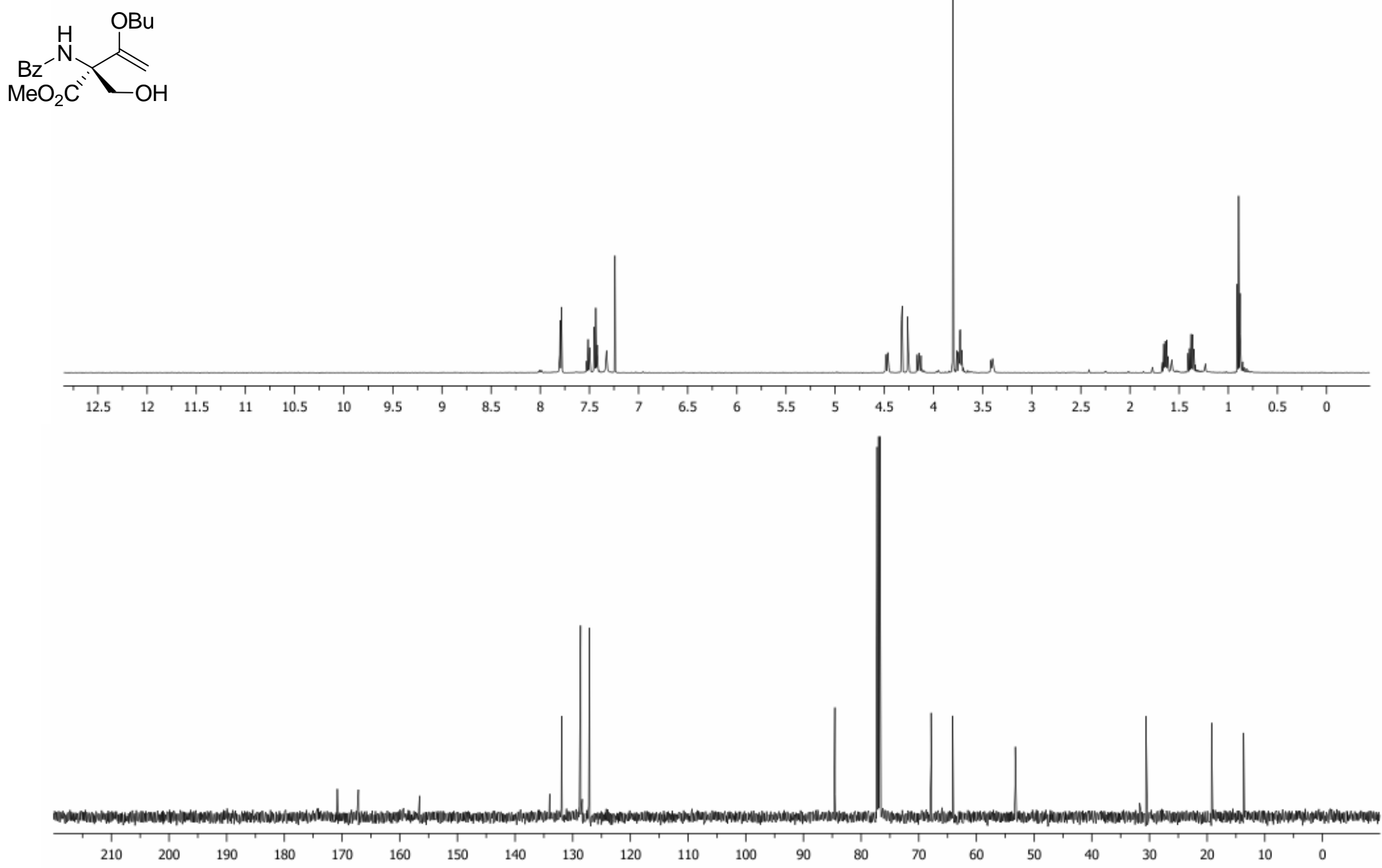
Methyl-2-(benzamido)-2-(hydroxymethyl)-3-methoxy-3-methylbutanoate(8)
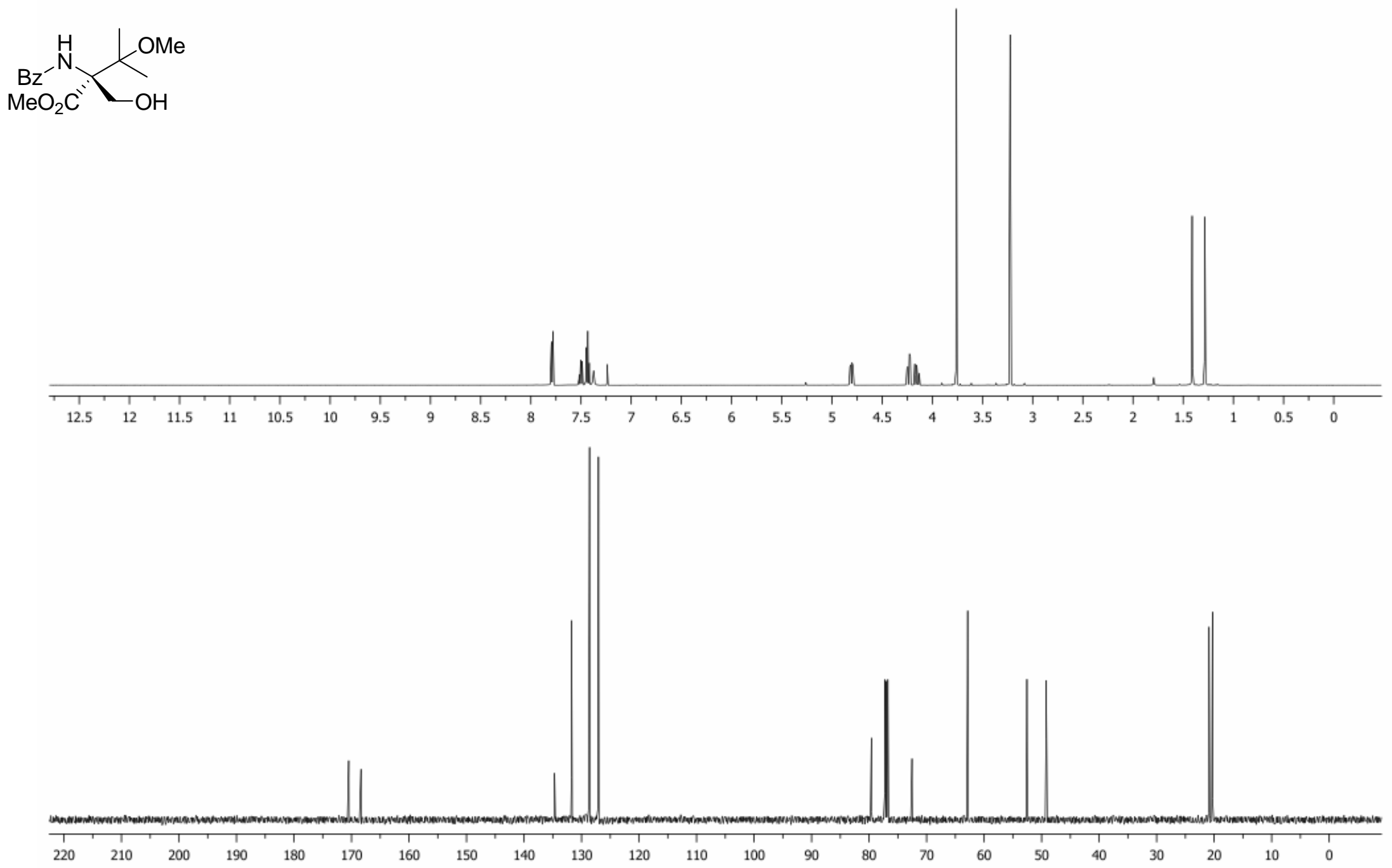
Methyl-2-(4-methoxybenzamido)-3-tert-butoxy-2-(hydroxymethyl)butanoate (10)

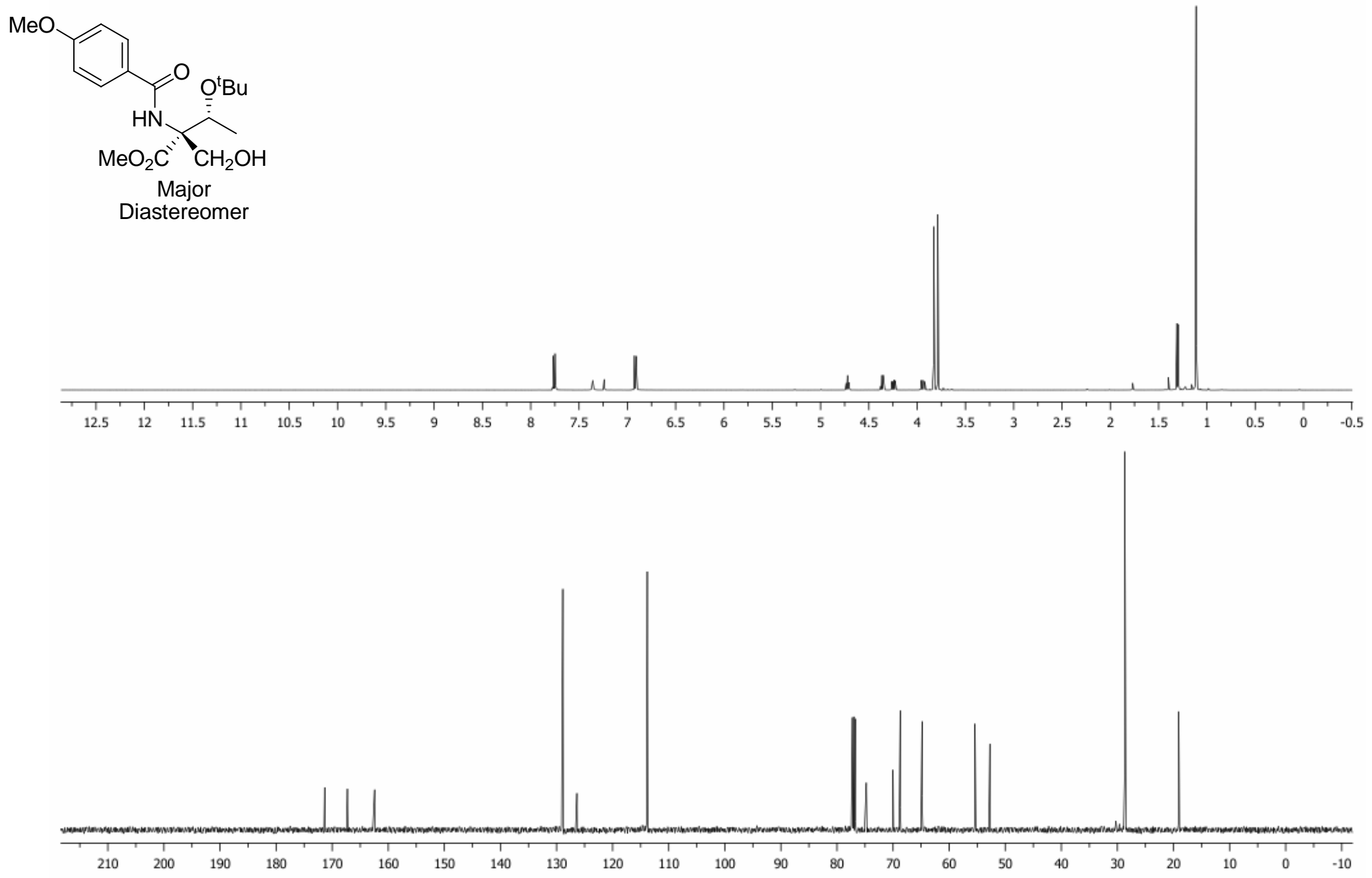


Methyl-2-(4-methoxybenzamido)-3-tert-butoxy-2-(hydroxymethyl)butanoate (10)

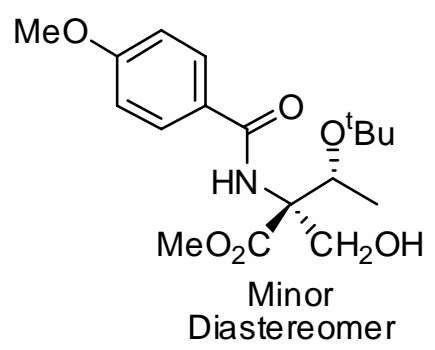

Diastereomer
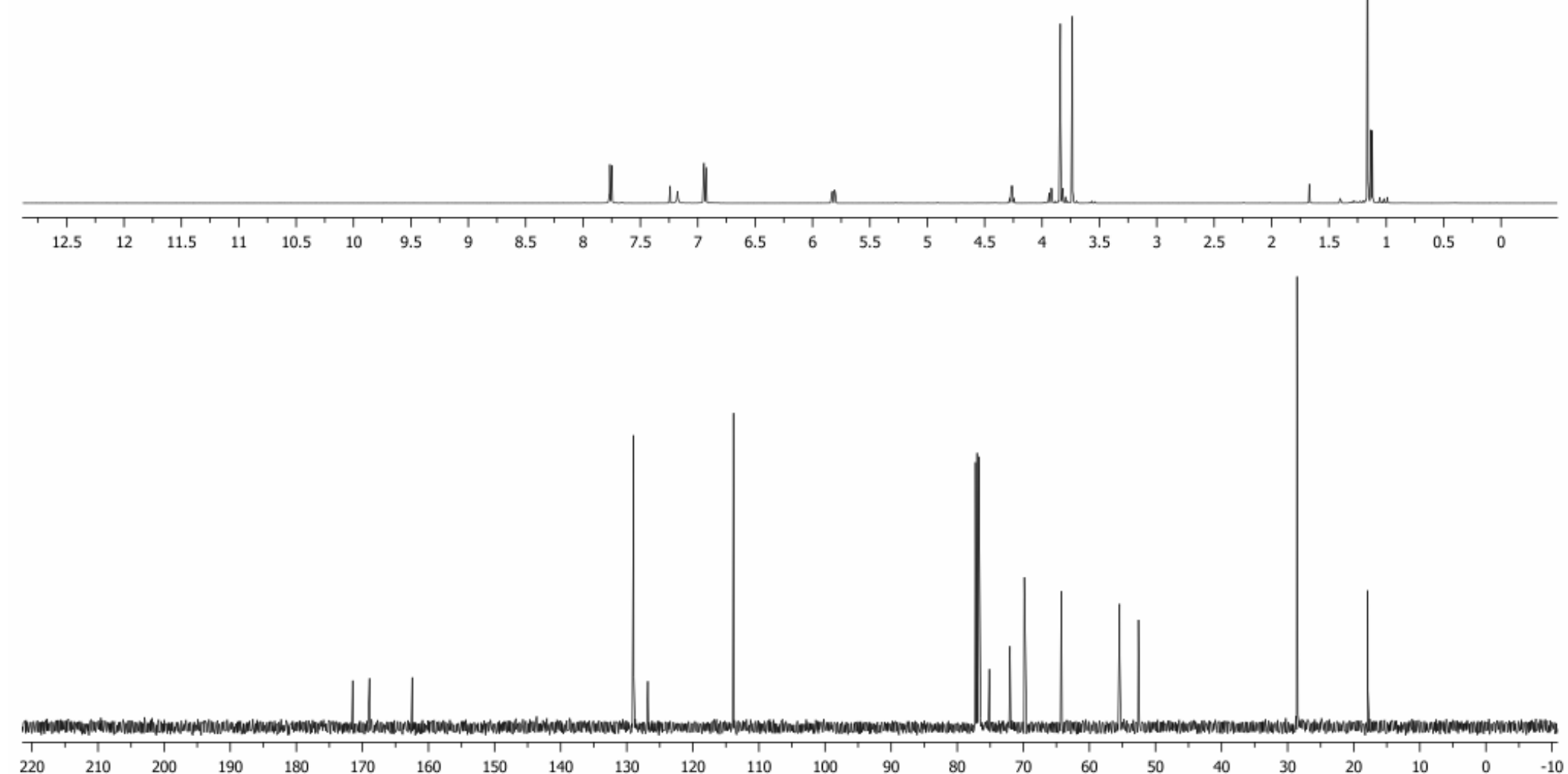
Methyl-2-(4-(trifluoromethyl)benzamido)-3-tert-butoxy-2-(hydroxymethyl) butanoate (11)
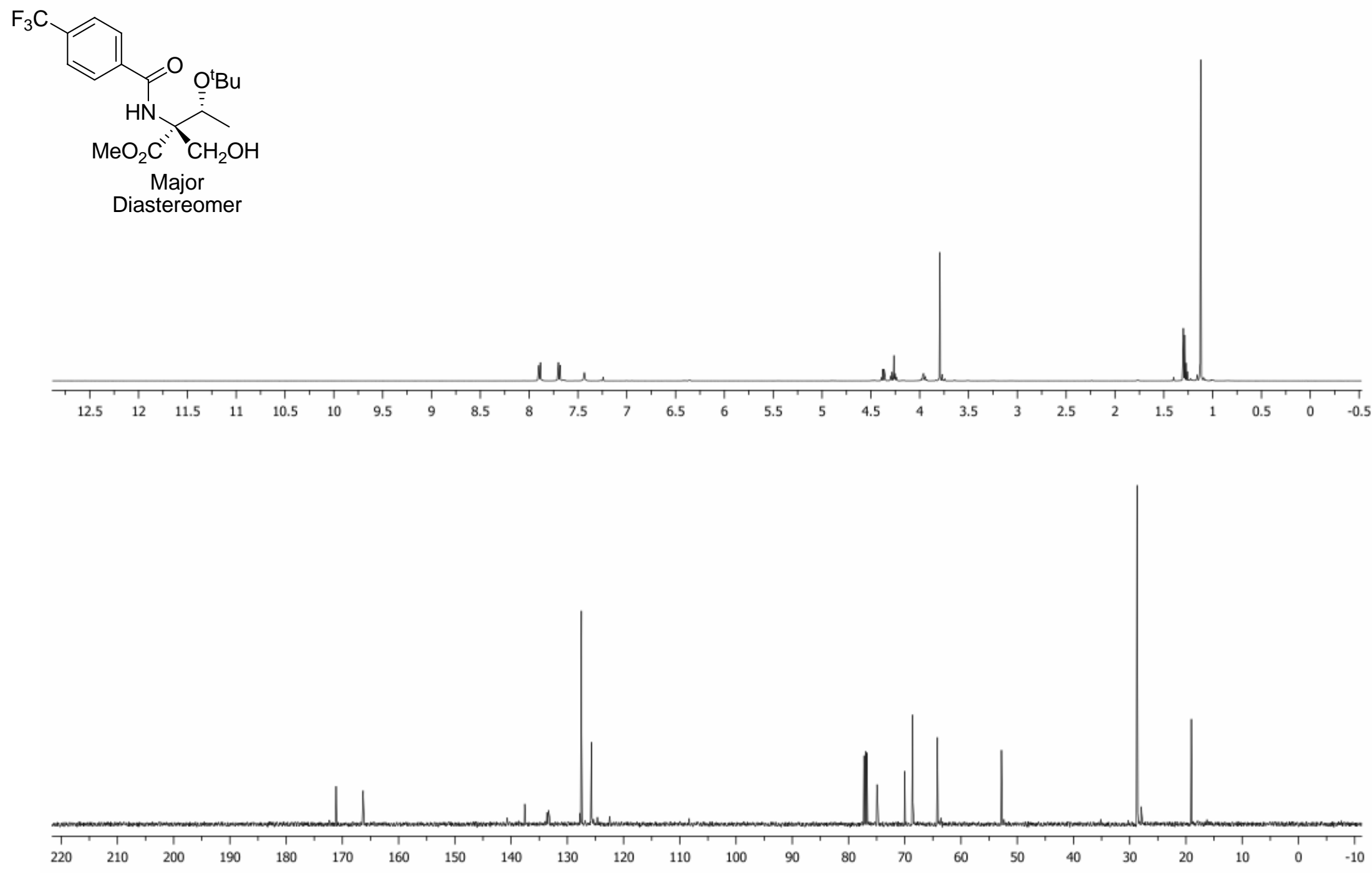
Methyl-2-(4-(trifluoromethyl)benzamido)-3-tert-butoxy-2-(hydroxymethyl) butanoate (11)
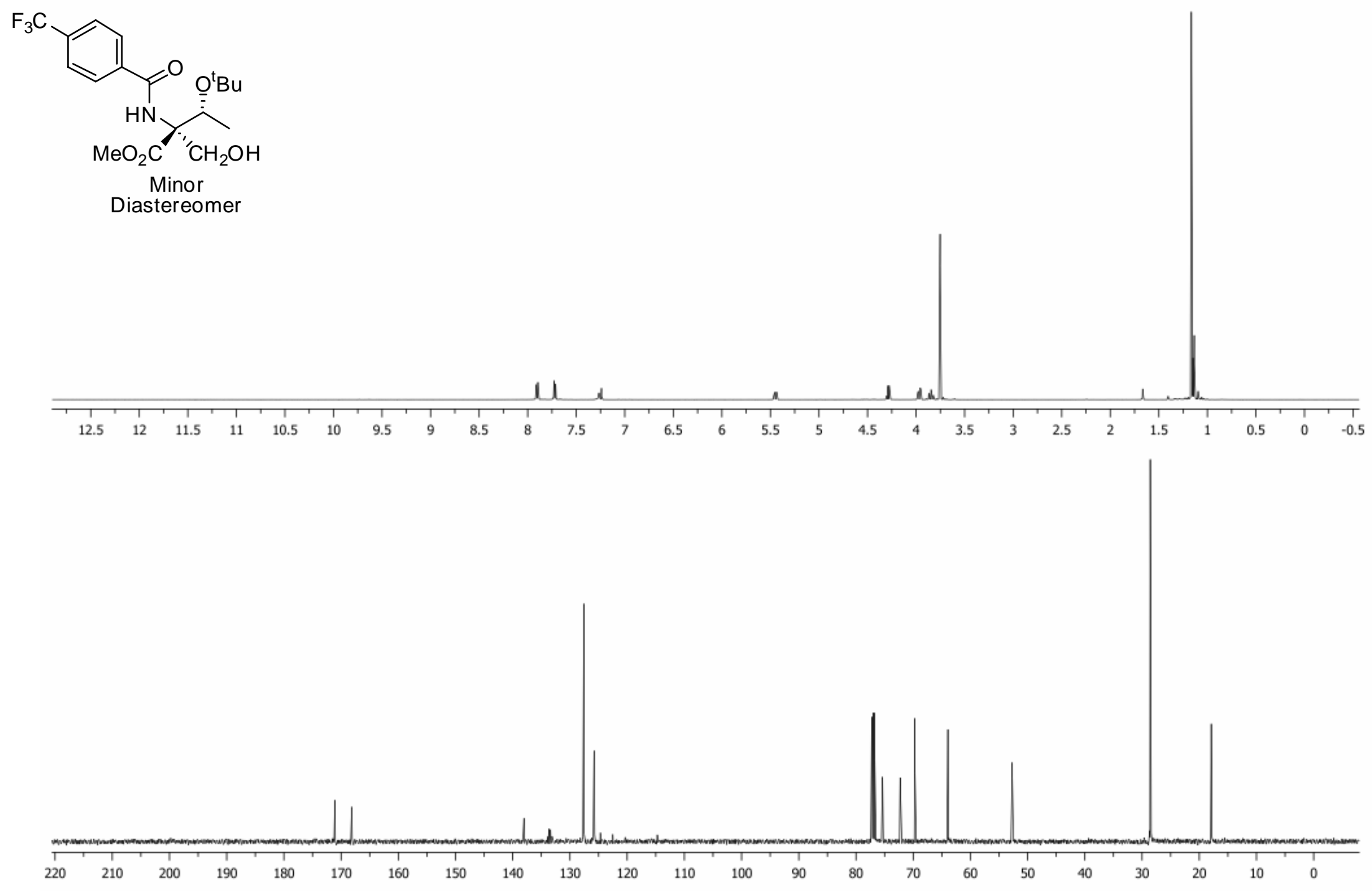
Methyl-3-tert-butoxy-2-(hydroxymethyl)-2-(propionamido)butanoate (12)

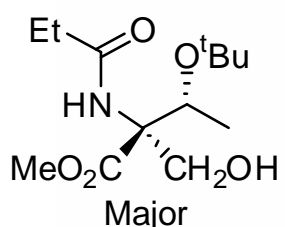

Diastereomer
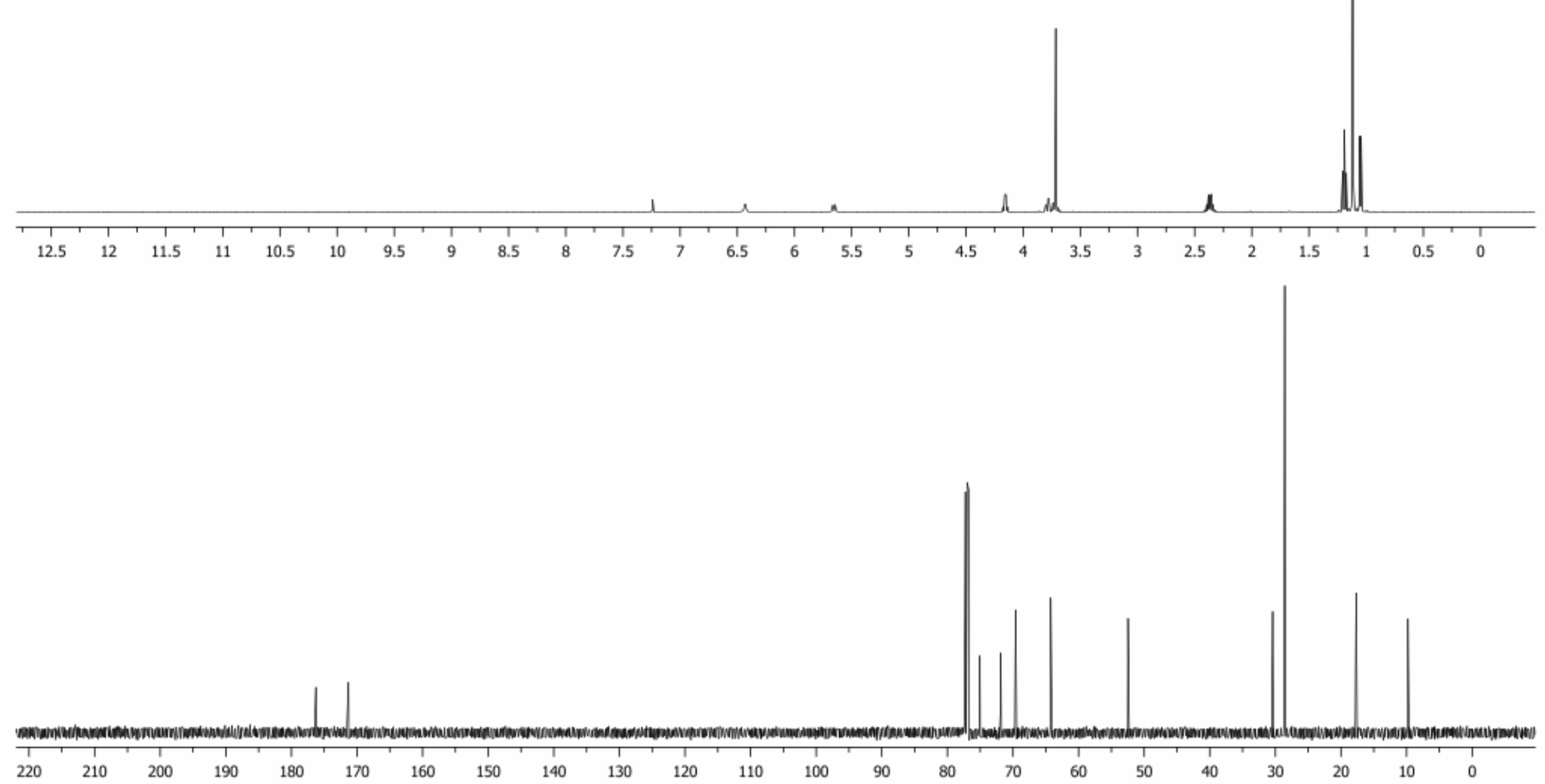
Methyl-3-tert-butoxy-2-(hydroxymethyl)-2-(propionamido)butanoate (12)
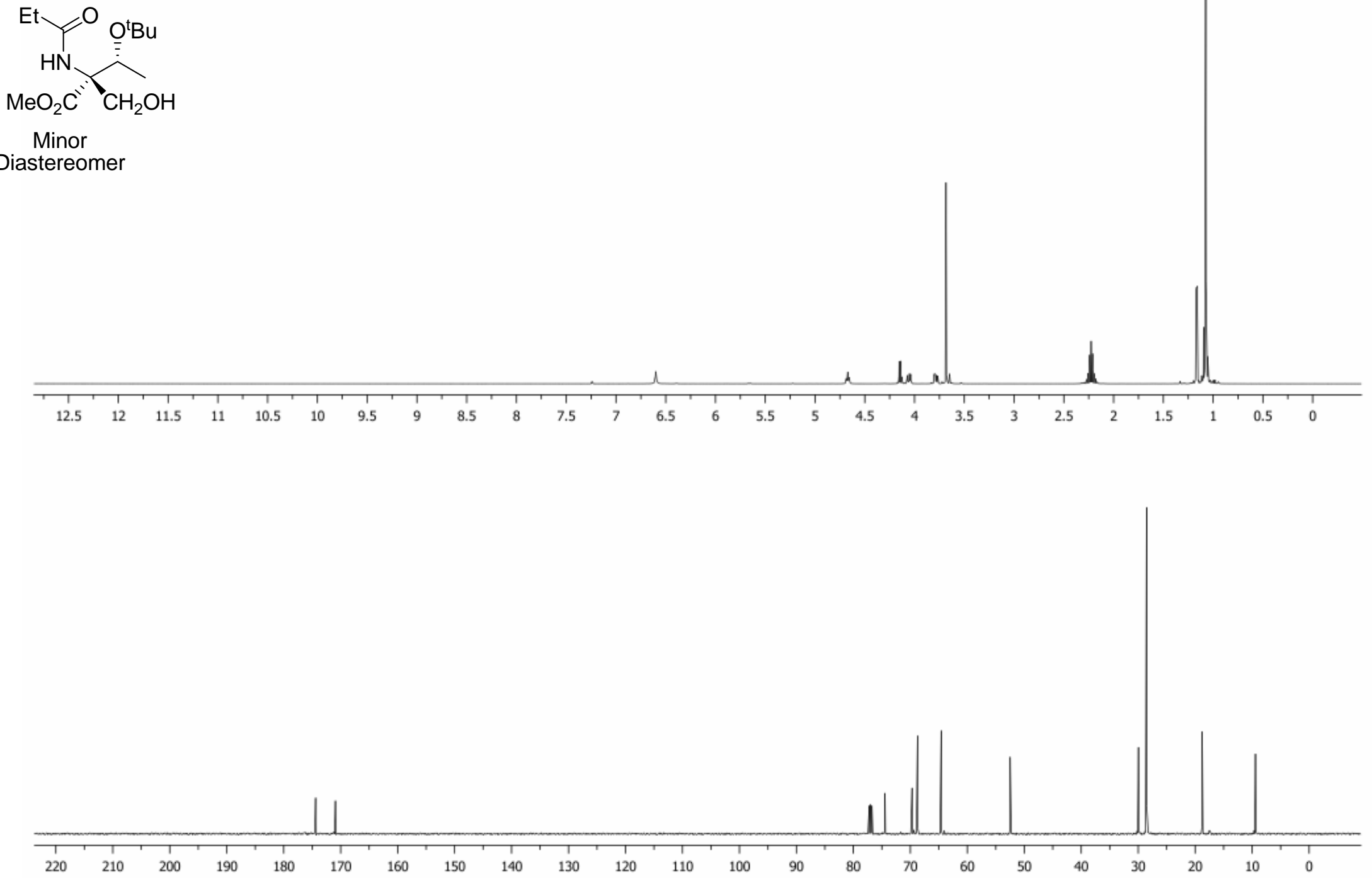
Methyl-2-(2-phenylacetamido)-3-tert-butoxy-2-(hydroxymethyl)butanoate (13)

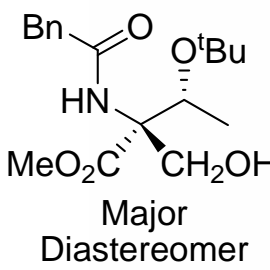

Diastereomer
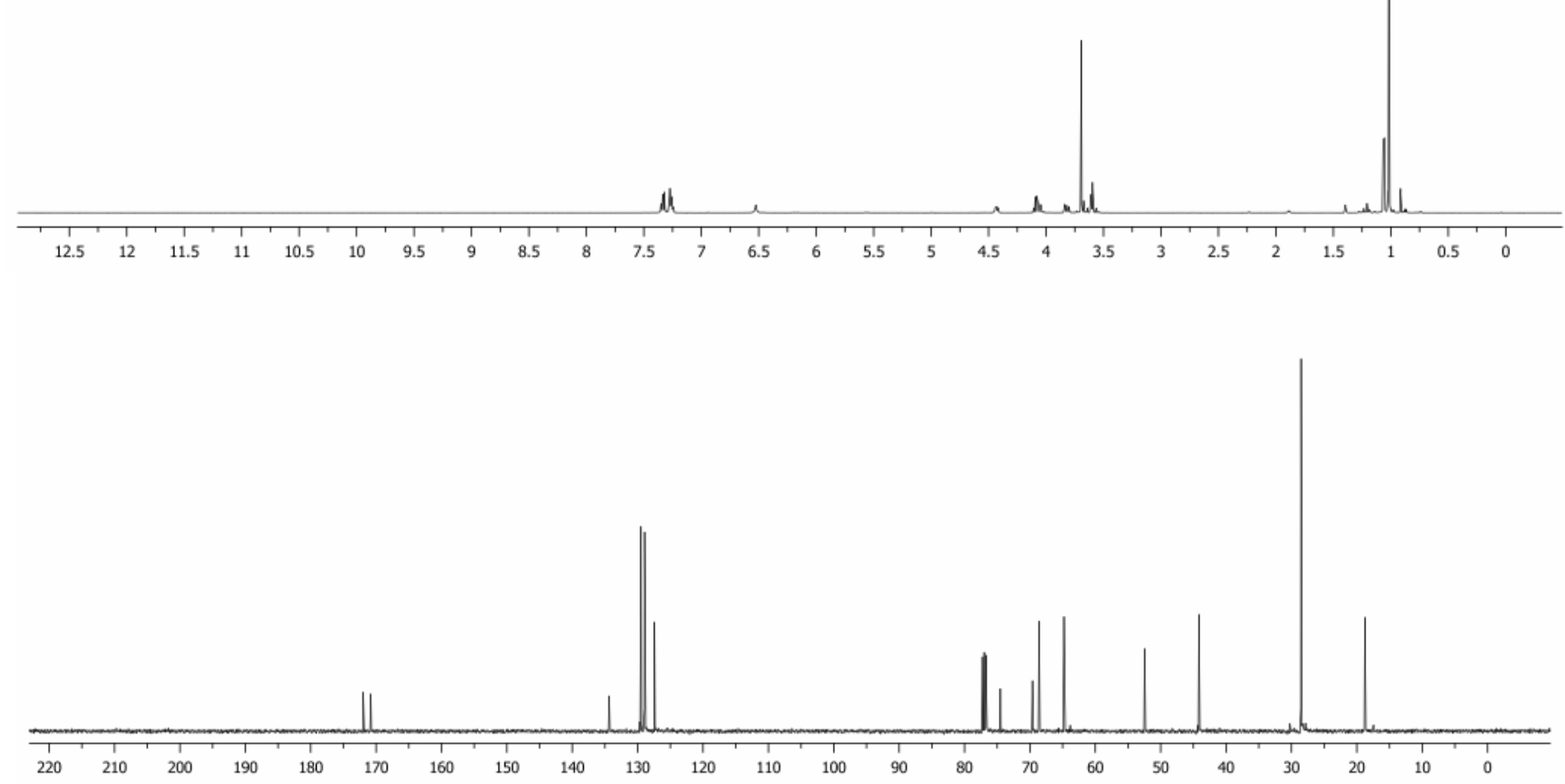
Methyl-2-(2-phenylacetamido)-3-tert-butoxy-2-(hydroxymethyl)butanoate (13)

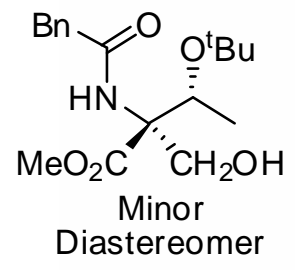

Diastereomer
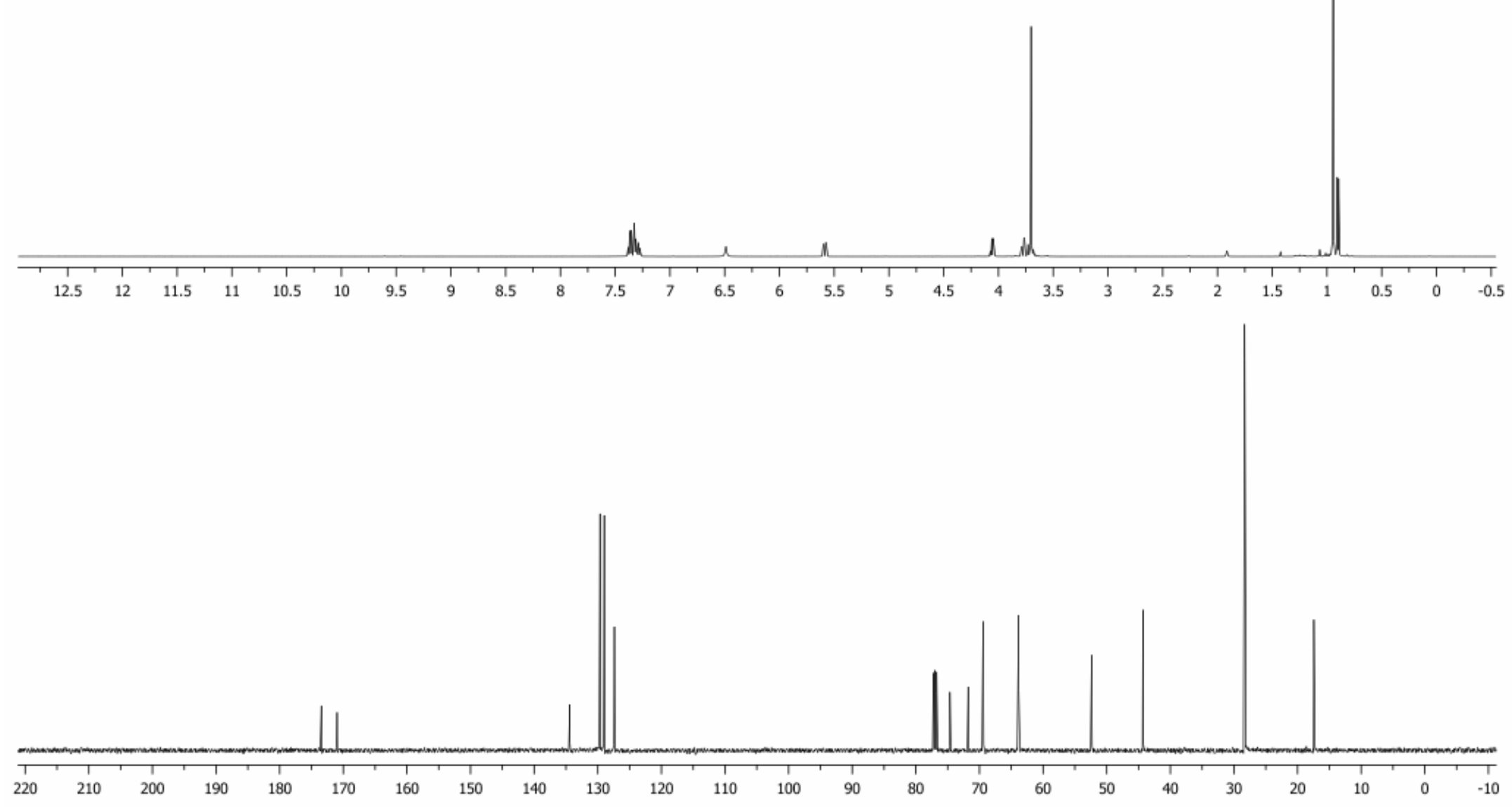
Methyl-5-(1-tert-butoxyethoxy)-2-ethyloxazole-4-carboxylate
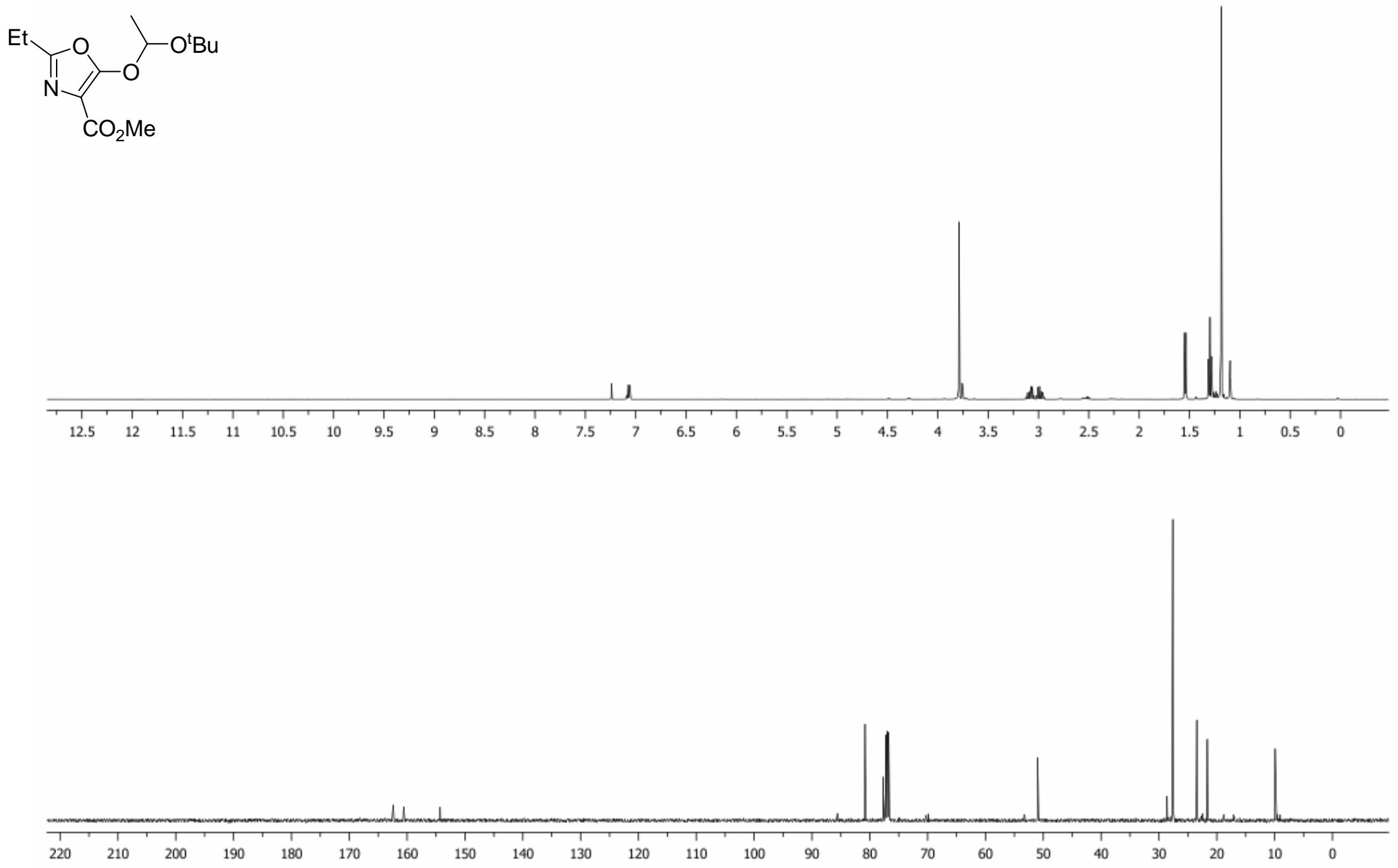
Methyl-4-(1-butoxy-2-deuterovinyl)-4,5-dihydro-5-oxo-2-phenyloxazole-4-carboxylate

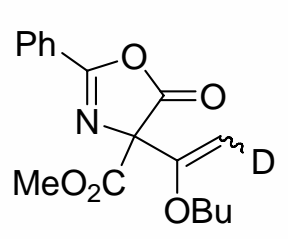

1:1 Mixture of Diastereomers
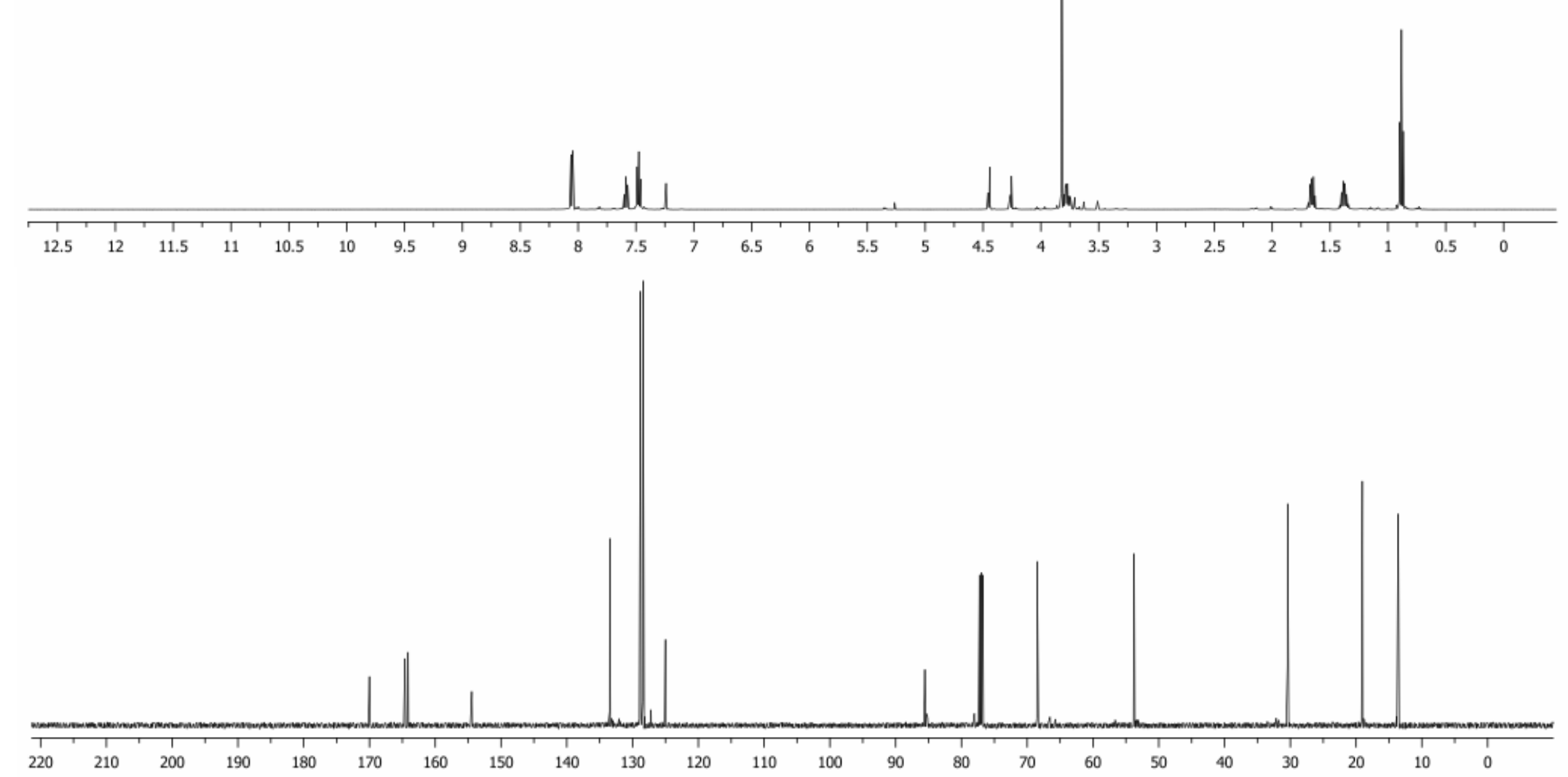